\title{
Quantum logic between remote quantum registers
}

\author{
N. Y. Yao, ${ }^{1}$ Z.-X. Gong, ${ }^{2}$ C. R. Laumann,,${ }^{1,3}$ S. D. Bennett, ${ }^{1}$ L.-M. Duan, ${ }^{2}$ M. D. Lukin, ${ }^{1}$ L. Jiang,,${ }^{4,5}$ and A. V. Gorshkov ${ }^{4}$ \\ ${ }^{1}$ Department of Physics, Harvard University, Cambridge, Massachusetts 02138, USA \\ ${ }^{2}$ Department of Physics and Michigan Center for Theoretical Physics, University of Michigan, Ann Arbor, Michigan 48109, USA \\ ${ }^{3}$ Institute for Theoretical Atomic Molecular and Optical Physics, Harvard University, Cambridge, Massachusetts 02138, USA \\ ${ }^{4}$ Institute for Quantum Information and Matter, California Institute of Technology, Pasadena, California 91125, USA \\ ${ }^{5}$ Department of Applied Physics, Yale University, New Haven, Connecticut 06511, USA
}

(Received 30 May 2012; revised manuscript received 15 December 2012; published 6 February 2013)

\begin{abstract}
We consider two approaches to dark-spin-mediated quantum computing in hybrid solid-state spin architectures. First, we review the notion of eigenmode-mediated unpolarized spin-chain state transfer and extend the analysis to various experimentally relevant imperfections: quenched disorder, dynamical decoherence, and uncompensated long-range coupling. In finite-length chains, the interplay between disorder-induced localization and decoherence yields a natural optimal channel fidelity, which we calculate. Long-range dipolar couplings induce a finite intrinsic lifetime for the mediating eigenmode; extensive numerical simulations of dipolar chains of lengths up to $L=12$ show remarkably high fidelity despite these decay processes. We further briefly consider the extension of the protocol to bosonic systems of coupled oscillators. Second, we introduce a quantum mirror based architecture for universal quantum computing that exploits all of the dark spins in the system as potential qubits. While this dramatically increases the number of qubits available, the composite operations required to manipulate dark-spin qubits significantly raise the error threshold for robust operation. Finally, we demonstrate that eigenmodemediated state transfer can enable robust long-range logic between spatially separated nitrogen-vacancy registers in diamond; disorder-averaged numerics confirm that high-fidelity gates are achievable even in the presence of moderate disorder.
\end{abstract}

DOI: 10.1103/PhysRevA.87.022306

PACS number(s): 03.67.Lx, 03.67.Hk, 05.50.+q, 75.10.Pq

\section{INTRODUCTION}

The ability to perform quantum logic between remote registers has emerged as a key challenge in the quest for scalable quantum architectures [1-4]. Qubits, the fundamental building blocks of such an architecture are often benchmarked by their coherence times [5-7]. Naturally, those qubit implementations that possess the longest coherence times also interact most weakly with their local environment, making multiqubit quantum logic in such systems difficult $[3,8]$. As a result, there has been tremendous recent interest in quantum data buses, which enable universal gates between physically separated quantum registers [9-16]. Such data buses have been proposed in systems ranging from trapped ions [17-19] and superconducting flux qubits [20-22] to coupled cavity arrays [23-25] and solid-state spin chains [26-40]. Prior proposals have focused on achieving perfect state transfer using either initialized [28,29,36,39], engineered [31,41,42], or dynamically controlled quantum channels [43-46].

By contrast, here we analyze a general method for high-fidelity quantum state transfer (QST) using an infinitetemperature (unpolarized) data bus as proposed in Refs. [3,27]. Our method requires neither external modulation during state transfer nor precisely engineered coupling strengths within the bus, making it an ideal candidate for solid-state spin-based quantum computing architectures $[3,26,47]$. We envision the long-range coherent interaction between remote qubits to be mediated by a specific collective eigenmode of the intermediate quantum data bus. In the solid state, such eigenmodes naturally suffer from localization effects associated with lattice imperfections and disorder [48]. Exploration of the interplay between such localization effects and intrinsic constraints set by finite coherence times is important to assess the feasibility of proposed architectures.
Our paper is organized as follows. In Sec. II we extend the previously proposed notion of eigenmode-mediated quantum state transfer [27] to the transverse field Ising model. In addition to being closely related to the actual achievable Hamiltonian of certain driven spin systems, this simple model enables an analytic description of the state-transfer protocol. In Sec. III we build upon these protocols and derive analytic expressions characterizing the channel fidelity for state transfer between remote quantum registers. Next we generalize our method to bosonic systems (e.g., coupled cavities and pendulum arrays) in Sec. IV. In particular, we demonstrate that chains of coupled oscillators can faithfully transport quantum information even at high oscillator temperature.

Having described eigenmode-mediated QST in both the fermionic and bosonic contexts, we then turn to a specific implementation within a solid-state quantum computing architecture. In Sec. V we analyze eigenmode-mediated quantum state transfer between remote spin quantum registers [49-51]. To be specific, we consider nitrogen-vacancy (NV) defect center registers and examine the optimization of state transfer fidelities in the presence of both disorder and a finite depolarization time $T_{1}$. The interplay between disorder-induced localization and decoherence yields a natural optimal channel fidelity, which we calculate. Ultimately, this optimization demonstrates the feasibility of scalable architectures whose remote logic gates can harbor infidelities below the threshold for error correcting codes [52,53]. While eigenmode-mediated QST fundamentally requires the register-chain coupling to be weaker than the intrachain coupling, in Sec. VI we consider generalizations to the strong-coupling regime. In particular, motivated by several recent studies $[18,39,54,55]$, we provide numerical simulations in parallel with the analytic channel fidelities derived in Sec. III. 
In Sec. VII we perform exact diagonalization for spin systems, which includes the full long-range dipolar interaction. We find remarkably high fidelities for our proposed QST protocols in chains of length up to $L=12$. Finally, in Sec. VIII we describe and analyze an alternate architecture, which utilizes globally controlled pulses for state transfer $[43,44]$. In this case we demonstrate that all spins in the system (e.g., even dark intermediate chain spins) can be viewed as potential qubits. However, while this dramatically increases the number of qubits available, the composite operations required to manipulate such intermediary spin qubits significantly raise the error threshold for robust operation.

\section{EIGENMODE-MEDIATED QST}

In this section we begin with an idealized system in which to understand eigenmode-mediated QST, namely, the transverse field Ising model

$$
H=-\sum_{i=1}^{N-1} \kappa \sigma_{i}^{x} \sigma_{i+1}^{x}+\sum_{i=1}^{N} B \sigma_{i}^{z},
$$

where $\kappa$ is the nearest-neighbor coupling strength and $B$ represents a uniform transverse field on each site. In addition to being realizable in a variety of experimental systems, ranging from NVs and trapped ions to electrons floating on helium [27,56,57], this model also has the virtue of being exactly solvable; this will allow us to clearly illustrate the essence of eigenmode-mediated state transfer and to understand the many-body entanglement that arises.

Expanding $\sigma_{i}^{x}$ as a function of spin-flip operators $\sigma_{i}^{ \pm}=$ $\left(\sigma_{i}^{x} \pm i \sigma_{i}^{y}\right) / 2$ and utilizing the Jordan-Wigner transformation [58] $c_{i}^{\dagger}=\sigma_{i}^{+} \exp \left(-i \pi \sum_{j=1}^{i-1} \sigma_{j}^{+} \sigma_{j}^{-}\right)$yields the fermionized Hamiltonian

$$
\begin{aligned}
H_{\mathrm{JW}}= & -\sum_{i=1}^{N-1} \kappa\left(c_{i}^{\dagger} c_{i+1}+c_{i}^{\dagger} c_{i+1}^{\dagger}-c_{i} c_{i+1}^{\dagger}-c_{i} c_{i+1}\right) \\
& +\sum_{i=1}^{N} B\left(c_{i}^{\dagger} c_{i}-c_{i} c_{i}^{\dagger}\right),
\end{aligned}
$$

which is quadratic and conserves fermionic parity without conserving particle number. To solve $H_{\mathrm{JW}}$, we reexpress it as $\vec{\phi}^{\dagger} A \vec{\phi}$, where we define $\vec{\phi}=\left(c_{1}, c_{2}, \ldots, c_{N}, c_{1}^{\dagger}, c_{2}^{\dagger}, \ldots, c_{N}^{\dagger}\right)^{T}$. The matrix $A$ is real and symmetric and is diagonalized to

$$
\Lambda=\left(\begin{array}{ccccc}
\epsilon_{1} & 0 & 0 & 0 & \ldots \\
0 & -\epsilon_{1} & 0 & 0 & \ldots \\
0 & 0 & \epsilon_{2} & 0 & \ldots \\
0 & 0 & 0 & -\epsilon_{2} & \ldots \\
\vdots & \vdots & \vdots & \vdots & \ddots
\end{array}\right)
$$

via an orthogonal matrix $\psi$ such that $\psi A \psi^{T}=\Lambda$. The eigenmodes come in pairs with energy $\pm \epsilon_{k}$, corresponding to eigenvectors $d_{k}=\psi_{2 k-1, j} \phi_{j}$ and $d_{k}^{\dagger}=\psi_{2 k, j} \phi_{j}$, where $k=$ $1, \ldots, N$. This transformation yields

$$
H_{\mathrm{JW}}=\sum_{k=1}^{N} \epsilon_{k}\left(d_{k}^{\dagger} d_{k}-d_{k} d_{k}^{\dagger}\right)
$$

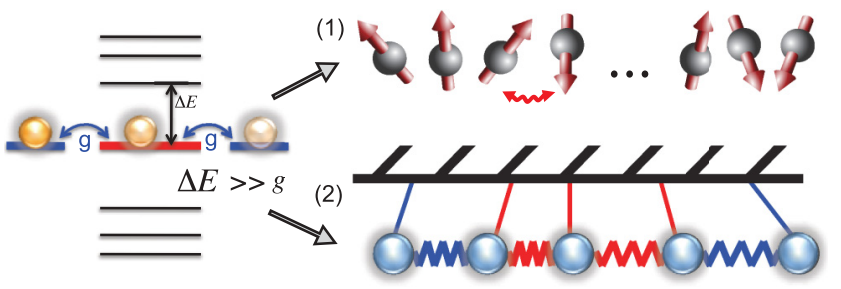

FIG. 1. (Color online) Quantum state transfer is achieved by tuning the left and right quantum registers (blue) to a particular eigenmode (red) of the intermediate data bus. By ensuring that the coupling $g$ between the registers and the chain is sufficiently weak relative to the spacing of adjacent eigenmodes, it is possible to consider evolution in an effective three-mode picture. Such eigenmode-mediated QST is applicable in a variety of contexts, ranging from solid-state spin chains to coupled bosonic degrees of freedom (e.g., pendulums or cavity arrays).

where the $d$ modes satisfy standard Dirac anticommutation relations. For a uniform chain the spectrum is $\epsilon_{k} \approx$ $\sqrt{\kappa^{2}+B^{2}-2 B \kappa \cos q_{k}}$, where $q_{k}=k \pi /(N+1)$.

We now consider the addition of quantum registers, labeled 0 and $N+1$, at the ends of the data bus (Fig. 1). The registers couple perturbatively with strength $g$ to the ends of the Ising spin chain $[27,28]$, and we apply a local Zeeman field $B^{\prime}$,

$$
H^{\prime}=-g\left(\sigma_{0}^{x} \sigma_{1}^{x}+\sigma_{N}^{x} \sigma_{N+1}^{x}\right)+B^{\prime}\left(\sigma_{0}^{z}+\sigma_{N+1}^{z}\right) .
$$

Upon fermionizing,

$$
\begin{aligned}
H_{\mathrm{JW}}^{\prime}= & -g\left(c_{0}^{\dagger} c_{1}+c_{0}^{\dagger} c_{1}^{\dagger}+c_{1}^{\dagger} c_{0}-c_{0} c_{1}\right) \\
& -g\left(c_{N}^{\dagger} c_{N+1}+c_{N}^{\dagger} c_{N+1}^{\dagger}+c_{N+1}^{\dagger} c_{N}-c_{N} c_{N+1}\right) \\
& +B^{\prime}\left(c_{0}^{\dagger} c_{0}-c_{0} c_{0}^{\dagger}+c_{N+1}^{\dagger} c_{N+1}-c_{N+1} c_{N+1}^{\dagger}\right)
\end{aligned}
$$

By tuning $B^{\prime}=\epsilon_{z}$ we ensure that the external registers are coupled resonantly to a single finite-energy eigenmode $d_{z}^{\dagger}$ of the intermediate chain. Quantum state transfer proceeds via resonant tunneling through this mode. Noting that

$$
c_{i}=\sum_{k=1}^{N}\left(\psi^{T}\right)_{i, 2 k-1} d_{k}+\sum_{k=1}^{N}\left(\psi^{T}\right)_{i, 2 k} d_{k}^{\dagger}
$$

allows us to reexpress $c_{1}$ and $c_{N}$ in terms of the $d$ modes. By choosing $g \psi_{2 z-1,1}=g \psi_{2 z-1, N} \ll B^{\prime},\left|\epsilon_{z}-\epsilon_{z \pm 1}\right|$ we ensure that off-resonance eigenmodes are only weakly coupled to the quantum registers, leaving an effective three-mode picture

$$
\begin{aligned}
H_{\mathrm{eff}} \approx & \epsilon_{z}\left(d_{z}^{\dagger} d_{z}-d_{z} d_{z}^{\dagger}\right)+\epsilon_{z}\left(c_{0}^{\dagger} c_{0}-c_{0} c_{0}^{\dagger}\right) \\
& +\epsilon_{z}\left(c_{N+1}^{\dagger} c_{N+1}-c_{N+1} c_{N+1}^{\dagger}\right)-g \psi_{2 z-1,1}\left(c_{0}^{\dagger} d_{z}+d_{z}^{\dagger} c_{0}\right) \\
& -g \psi_{2 z-1, N}\left(c_{N+1}^{\dagger} d_{z}+d_{z}^{\dagger} c_{N+1}\right) .
\end{aligned}
$$

It is interesting to note that for $B<\kappa$, the Hamiltonian in Eq. (2) represents a spinless $p$-wave superconductor in its topological phase [59]. The zero-energy boundary modes of this system have received a great deal of attention recently. As these Majorana zero modes are exponentially localized [60], they cannot be employed for state transfer. In our analysis, this follows from the failure of the secular approximation to remove fermion number nonconserving terms when $B^{\prime}=\epsilon_{z}=$ 0 . A straightforward calculation shows that the pairing terms precisely cancel the hopping terms in the effective evolution. 


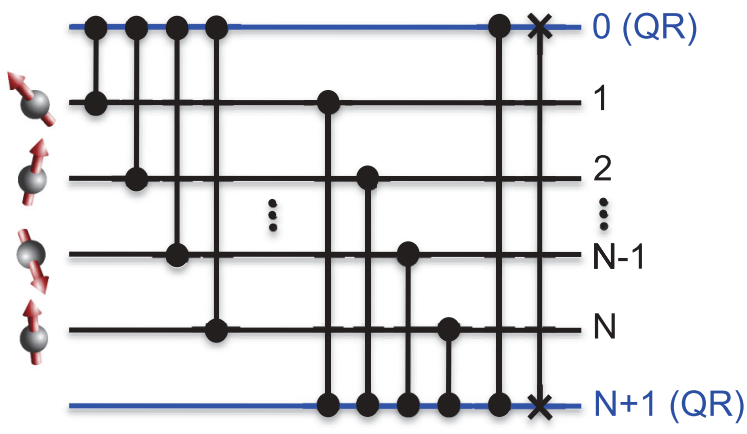

FIG. 2. (Color online) Schematic circuit diagram depicting eigenmode-mediated state transfer between the quantum registers (QR) 0 and $N+1$. Controlled-PHASE gates are represented as circle-ending dumbbells while cross-ending dumbbells depict a SWAP gate. In addition to the desired state transfer, each register is $\mathrm{CP}$ entangled with all intermediate spins owing to the Wigner strings associated with fermionization. This additional entanglement can be canceled by utilizing a simple two-qubit encoding.

Equation (7) represents the key result of the above manipulations. State transfer is achieved by time evolving for $\tau=\frac{\pi}{\sqrt{2} g \psi_{2 z-1,1}}$, leading to unitary evolution

$$
\begin{aligned}
U_{\mathrm{eff}} & =e^{-i \tau H_{\mathrm{eff}}}=(-1)^{n_{z}}(-1)^{\left(c_{0}^{\dagger}+c_{N+1}^{\dagger}\right)\left(c_{0}+c_{N+1}\right) / 2} \\
& =(-1)^{n_{z}}\left[1-\left(c_{0}^{\dagger}+c_{N+1}^{\dagger}\right)\left(c_{0}+c_{N+1}\right)\right],
\end{aligned}
$$

where $n_{z}=d_{z}^{\dagger} d_{z}$. It is instructive to write the explicit action of $U_{\text {eff }}$ on the subspace spanned by $\Psi=$ $\left\{|\Omega\rangle, c_{0}^{\dagger}|\Omega\rangle, c_{N+1}^{\dagger}|\Omega\rangle, c_{0}^{\dagger} c_{N+1}^{\dagger}|\Omega\rangle\right\}$, where $|\Omega\rangle$ is the vacuum associated with $c_{0}, c_{N+1}$,

$$
U_{\text {eff }} \Psi=(-1)^{n_{z}}\left(\begin{array}{cccc}
1 & 0 & 0 & 0 \\
0 & 0 & -1 & 0 \\
0 & -1 & 0 & 0 \\
0 & 0 & 0 & -1
\end{array}\right) \Psi
$$

Up to signs, the effective evolution in the register subspace is a SWAP gate. In the spin representation, owing to Wigner strings $\left[\exp \left(-i \pi \sum_{j=1}^{i-1} \sigma_{j}^{+} \sigma_{j}^{-}\right)\right]$, there exists an additional set of controlled-PHASE (CP) gates, as shown in Fig. 2. Since $\mathrm{CP}^{2}=$ $\mathbb{I}$, this entanglement can be easily canceled and logic gates between the remote registers can be successfully accomplished $[47,61]$. We detail two possible such protocols below.

The first protocol that we consider is particularly applicable to the case of multiqubit quantum registers. For a two-qubit register, we can label one qubit as the memory qubit while the other represents the coupling qubit. Once an eigenmodemediated swap between the coupling qubits is accomplished, an intraregister $\mathrm{CP}$ gate is then performed between the two qubits of the remote register. The return swap then cancels the unwanted entanglement illustrated in Fig. 2, leaving only a controlled-PHASE gate between the two memory qubits. Since $\mathrm{CP}$ gates are themselves universal, such a procedure enables universal logic between remote registers.

An alternate method, which we call the paired protocol, utilizes a two-qubit encoding to cancel the Wigner strings. In this approach, the quantum information is encoded in two spins $a$ and $b$ with logical basis $|\downarrow\rangle=|\downarrow\rangle_{a}|\downarrow\rangle_{b},|\uparrow\rangle=|\uparrow\rangle_{a}|\uparrow\rangle_{b}$
$[27,47,61]$; the intuition behind this encoding is that it produces an effective bosonic excitation, thereby mitigating the effect of the fermionic Wigner strings. State transfer proceeds by successively transferring $a$ and $b$ through the intermediary chain.

\section{ANALYTIC CHANNEL FIDELITY}

We now derive the channel fidelity associated with the paired protocol. To set up the analytic framework, we begin by calculating the fidelity of a simplified protocol, termed the double swap. In this double swap, we consider the left register (indexed 0) undergoing two successive eigenmodemediated SWAP gates. Ideally, this simplified protocol swaps the quantum information twice, thereby disentangling it from the intermediate chain and also returning it to its initial position at the left register. We then consider a second protocol, termed the single swap, in which the quantum information undergoes only one eigenmode-mediated SWAP gate. Analyzing this protocol will illustrate the effect of the residual entanglement on the channel fidelity. Finally, we turn to the paired protocol and demonstrate that the proposed two-qubit encoding can eliminate this entanglement, thereby enabling quantum state transfer.

\section{A. Double swap}

The average channel fidelity for a quantum dynamical operation is given by

$$
F=\frac{1}{2}+\frac{1}{12} \sum_{i=x, y, z} \operatorname{Tr}\left[\sigma^{i} \mathcal{E}\left(\sigma^{i}\right)\right],
$$

where $\mathcal{E}$ characterizes the quantum channel [62]. For simplicity of notation, we will restrict ourselves to the $X X$ model $H=$ $g\left(\sigma_{0}^{+} \sigma_{1}^{-}+\sigma_{N}^{+} \sigma_{N+1}^{-}+\right.$H.c. $)+\sum_{i=1}^{N-1} \kappa\left(\sigma_{i}^{+} \sigma_{i+1}^{-}+\right.$H.c. $), \quad$ although analogous results hold for the previously considered transverse field Ising model. For the double swap (DS), we let $U$ represent evolution under $H$ for a time $t=2 \tau$ equivalent to twice the state-transfer time. Let us suppose that the left register is initially disentangled from the remainder of the chain, which is in a thermal mixed state $\rho_{\mathrm{ch}}^{\mathrm{DS}}$; the average double-swap channel fidelity is then given by

$$
\begin{aligned}
F_{\mathrm{DS}} & =\frac{1}{2}+\frac{1}{12} \sum_{i=x, y, z} \operatorname{Tr}\left[\sigma_{0}^{i} U\left(\sigma_{0}^{i} \otimes \rho_{\mathrm{ch}}^{\mathrm{DS}}\right) U^{\dagger}\right] \\
& =\frac{1}{2}+\frac{1}{12} \sum_{i=x, y, z} \operatorname{Tr}\left[U^{\dagger} \sigma_{0}^{i} U\left(\sigma_{0}^{i} \otimes \rho_{\mathrm{ch}}^{\mathrm{DS}}\right)\right] \\
& =\frac{1}{2}+\frac{1}{12} \sum_{i=x, y, z} \operatorname{Tr}\left[\sigma_{0}^{i}(t)\left(\sigma_{0}^{i} \otimes \rho_{\mathrm{ch}}^{\mathrm{DS}}\right)\right],
\end{aligned}
$$

where $\sigma_{0}^{i}(t)$ is the Heisenberg evolution of the left register. By fermionization, this evolution can be reexpressed with respect to elements of the matrix $M=e^{-i K t}$, where $K$ is the $(N+2) \times(N+2)$ coupling matrix of the full Hamiltonian (including registers) $H=\sum_{i, j=0}^{N+1} K_{i j} c_{i}^{\dagger} c_{j}$. Evolution of the Fermi operators is governed by $\dot{c}_{m}=-i \sum_{n} K_{m n} c_{n}$, implying 
that $c_{m}(t)=\sum_{n} M_{m n} c_{n}$ and further that

$$
\begin{aligned}
\sigma_{0}^{+}(t) & =U^{\dagger} \sigma_{0}^{+} U=U^{\dagger} c_{0}^{\dagger} U \\
& =\sum_{i} M_{0 i}^{*} c_{i}^{\dagger}=\sum_{i} M_{0 i}^{*} \sigma_{i}^{+} \prod_{l<i} e^{i \pi \sigma_{l}^{+} \sigma_{l}^{-}}, \\
\sigma_{0}^{z}(t)= & 2 c_{0}^{\dagger}(t) c_{0}(t)-1 \\
= & -1+2 \sum_{i j} M_{0 i}^{*} M_{0 j} c_{i}^{\dagger} c_{j} \\
= & -1+2 \sum_{i j} M_{0 i}^{*} M_{0 j} \sigma_{i}^{+} \sigma_{j}^{-} \prod_{i<l<j} e^{i \pi \sigma_{l}^{+} \sigma_{l}^{-}},
\end{aligned}
$$

where we have used the fact that $c_{0}^{\dagger}$ carries no Wigner string. To evaluate $F_{\mathrm{DS}}$, we note that $\sigma^{ \pm}=\left(\sigma^{x} \pm i \sigma^{y}\right) / 2$ and hence $\operatorname{Tr}\left[\sigma_{0}^{x}(t)\left(\sigma_{0}^{x} \otimes \rho_{\mathrm{ch}}\right)\right]=\operatorname{Tr}\left\{\left[\sigma_{0}^{+}(t)+\sigma_{0}^{-}(t)\right]\left[\left(\sigma_{0}^{+}+\right.\right.\right.$ $\left.\left.\left.\sigma_{0}^{-}\right) \otimes \rho_{\mathrm{ch}}\right]\right\}$. Contributions are only obtained from the cross terms $\sigma_{0}^{+}(t)\left(\sigma_{0}^{-} \otimes \rho_{\mathrm{ch}}\right)$ and $\sigma_{0}^{-}(t)\left(\sigma_{0}^{+} \otimes \rho_{\mathrm{ch}}\right)$ since the number of excitations in $i=0$ must be preserved to generate a nonzero trace. For example, using Eq. (12),

$$
\begin{aligned}
\operatorname{Tr} & {\left[\sigma_{0}^{+}(t)\left(\sigma_{0}^{-} \otimes \rho_{\mathrm{ch}}\right)\right] } \\
& =\operatorname{Tr}\left[\left(\sum_{i} M_{0 i}^{*} \sigma_{i}^{+} \prod_{l<i} e^{i \pi \sigma_{l}^{+} \sigma_{l}^{-}}\right)\left(\sigma_{0}^{-} \otimes \rho_{\mathrm{ch}}\right)\right] \\
& =\operatorname{Tr}\left[M_{00}^{*} \sigma_{0}^{+} \sigma_{0}^{-} \otimes \rho_{\mathrm{ch}}\right]=M_{00}^{*} .
\end{aligned}
$$

An analogous calculation yields $\operatorname{Tr}\left[\sigma_{0}^{-}(t)\left(\sigma_{0}^{+} \otimes \rho_{\mathrm{ch}}\right)\right]=M_{00}$. Finally, for the $\sigma^{z}$ terms, one finds, using Eq. (13),

$$
\begin{aligned}
\operatorname{Tr}\left[\sigma_{0}^{z}(t)\left(\sigma_{0}^{z} \otimes \rho_{\mathrm{ch}}\right)\right] & =\operatorname{Tr}\left[-\sigma_{0}^{z} \otimes \rho_{\mathrm{ch}}\right]+\operatorname{Tr}\left[\left(2 \sum_{i j} M_{0 i}^{*} M_{0 j} \sigma_{i}^{+} \sigma_{j}^{-} \prod_{i<l<j} e^{i \pi \sigma_{l}^{+} \sigma_{l}^{-}}\right)\left(\sigma_{0}^{z} \otimes \rho_{\mathrm{ch}}\right)\right] \\
& =\operatorname{Tr}\left[2 M_{00}^{*} M_{00} \sigma_{0}^{+} \sigma_{0}^{-} \sigma_{0}^{z} \otimes \rho_{\mathrm{ch}}\right]=2\left|M_{00}\right|^{2},
\end{aligned}
$$

where we have noted that $i=j$ to ensure that the number of excitations in each mode is conserved. Moreover, we must also have that $i=j=0$, since $\operatorname{Tr}\left[\sigma_{0}^{z}\right]=0$. Combining the above terms yields the double-swap channel fidelity as

$$
F_{\mathrm{DS}}=\frac{1}{2}+\frac{1}{6}\left(M_{00}+M_{00}^{*}+\left|M_{00}\right|^{2}\right) .
$$

Interestingly, we need to compute only a single matrix element to obtain the relevant channel fidelity.

\section{B. Single swap}

We now consider the single-swap (SS) channel fidelity associated with the transfer of quantum information from the right register (indexed $N+1$ ) to the left register (indexed 0 )

$$
F_{\mathrm{SS}}=\frac{1}{2}+\frac{1}{12} \sum_{i=x, y, z} \operatorname{Tr}\left[\sigma_{0}^{i}(t)\left(\rho_{\mathrm{ch}}^{\mathrm{SS}} \otimes \sigma_{N+1}^{i}\right)\right]
$$

where $\rho_{\text {ch }}^{\text {SS }}$ now characterizes the initial state for spins $\{0, \ldots, N\}$. Note that $F_{\mathrm{SS}}$ will be independent of the direction of state transfer and we have chosen right to left for notational simplicity. From Eq. (12) one finds

$$
\begin{aligned}
\sigma_{0}^{x}(t) & =c_{0}^{\dagger}(t)+c_{0}(t)=\sum_{i} M_{0 i}^{*} c_{i}^{\dagger}+M_{0 i} c_{i} \\
& =\sum_{i}\left[\left\{\operatorname{Re}\left(M_{0 i}\right) \sigma_{i}^{x}+\operatorname{Im}\left(M_{0 i}\right) \sigma_{i}^{y}\right\} \prod_{l=0}^{i-1}\left(-\sigma_{l}^{z}\right)\right] .
\end{aligned}
$$

In analogy to the DS case, $i \neq N+1$ terms do not contribute to the trace

$$
\operatorname{Tr}\left[\sigma_{0}^{x}(t)\left(\rho_{\mathrm{ch}} \otimes \sigma_{N+1}^{x}\right)\right]=2 \operatorname{Re}\left(M_{0, N+1}\right) \operatorname{Tr}\left[\rho_{\mathrm{ch}}^{\mathrm{sS}} \prod_{l=0}^{N}\left(-\sigma_{l}^{z}\right)\right] .
$$

The $\sigma^{y}$ term yields an identical contribution while the $\sigma^{z}$ term yields $\operatorname{Tr}\left[\sigma_{0}^{z}(t)\left(\rho_{\mathrm{ch}}^{\mathrm{SS}} \otimes \sigma_{N+1}^{z}\right)\right]=2\left|M_{0, N+1}\right|^{2}$. Therefore,

$$
\begin{aligned}
F_{\mathrm{SS}}= & \frac{1}{2}+\frac{1}{6}\left(2 \operatorname{Re}\left(M_{0, N+1}\right) \operatorname{Tr}\left[\rho_{\mathrm{ch}}^{\mathrm{SS}} \prod_{l=0}^{N}\left(-\sigma_{l}^{z}\right)\right]\right. \\
& \left.+\left|M_{0, N+1}\right|^{2}\right) .
\end{aligned}
$$

For perfect transfer with $F_{\mathrm{SS}}=1$, we would require both $\left|M_{0, N+1}\right|=1$ and $\left|\operatorname{Tr}\left[\rho_{\mathrm{ch}}^{\mathrm{SS}} \prod_{l=0}^{N}\left(-\sigma_{l}^{z}\right)\right]\right|=1$. In the case of an unpolarized chain, the second condition is unsatisfied since the expectation value of the chain parity operator $P=\prod_{l=0}^{N}\left(-\sigma_{l}^{z}\right)$ is zero. The dependence of the single-swap fidelity on the intermediate chain's parity demonstrates the entanglement illustrated in Fig. 2 and presents an obvious problem for QST.

\section{Paired protocol}

To overcome this problem, we now turn to the two-qubit encoding proposed in Sec. II, e.g., $|\downarrow\rangle=|\downarrow\rangle_{a}|\downarrow\rangle_{b},|\uparrow\rangle=$ $|\uparrow\rangle_{a}|\uparrow\rangle_{b}$. Let us index the full chain as $\left\{0_{a}, 0_{b}, 1, \ldots, N,(N+\right.$ $\left.1)_{b},(N+1)_{a}\right\}$ and define $U_{b}$ as the transfer process through the subchain $\left\{0_{b}, 1, \ldots, N,(N+1)_{b}\right\}$, while $U_{a}$ represents the transfer process through the subchain $\left\{0_{a}, 1, \ldots, N,(N+\right.$ $1)_{a}$ \}. The composite $U=U_{b} U_{a}$ then represents the unitary characterizing the encoded state transfer, with average channel fidelity given by

$$
F_{\text {enc }}=\frac{1}{2}+\frac{1}{12} \sum_{i=x, y, z} \operatorname{Tr}\left[\sigma_{N+1}^{i}(t)\left(\sigma_{0}^{i} \otimes \rho_{\mathrm{ch}}^{\mathrm{PP}} \otimes \rho_{N+1}\right)\right] .
$$

Here $\rho_{\mathrm{ch}}^{\mathrm{PP}}$ is the mixed initial state of the intermediate chain $(\{1, \ldots, N\}), \sigma_{0}^{i}$ acts on the encoded logical subspace of the 0 register, and $\rho_{N+1}$ is the mixed state of the encoded $(N+1)$ register within the logical subspace. Working within this logical subspace is crucial to ensure that $\mathrm{CP}_{0_{a}, N+1_{a}} \mathrm{CP}_{0_{b}, N+1_{b}}=$ II. Inspection reveals that the paired protocol includes two 
contributions from the chain parity operator and since $P^{2}=$ II, we have effectively disentangled from the intermediate chain. Since a consistent ordering of the spin chain is required to implement the Jordan-Wigner transformation, the Hamiltonian $H_{U_{a}}$ governing the $U_{a}$ transfer evolution will contain uncanceled Wigner strings. For example, the piece of $H_{U_{a}}$ containing the coupling between the registers and the ends of the spin chain takes the form $H_{U_{a}}=$ $g\left(c_{0_{a}}^{\dagger} e^{i \pi n_{0_{b}}} c_{1}+c_{N}^{\dagger} e^{i \pi n_{(N+1)_{b}}} c_{(N+1)_{a}}+\right.$ H.c. $)$. While one must take care to correctly evaluate such strings, an otherwise straightforward computation yields

$$
\begin{aligned}
F_{\text {enc }}= & \frac{1}{6}\left(2\left|M_{0, N+1}\right|^{2} \operatorname{Re}\left[M_{0, N+1}^{2}-M_{0,0} M_{N+1, N+1}\right]\right. \\
& \left.+\left|M_{0, N+1}\right|^{2}+\left|\sum_{i} M_{N+1, i} M_{i, 0}\right|^{2}\right)+\frac{1}{2} .
\end{aligned}
$$

Again, one only needs to compute certain matrix elements of $M$ and in fact an analytic form for all such elements can be obtained (see Appendixes A and B for details) [28].

\section{GENERALIZATION TO OSCILLATOR SYSTEMS}

Motivated by the fact that quadratic bosonic systems can also be exactly solved and by the tremendous experimental progress in realizing coupled-oscillator arrays, in this section we analyze the generalization of eigenmode-mediated state transfer to systems of bosonic oscillators. By contrast to our previous discussions of dipolar spin chains, where we made an explicit nearest-neighbor assumption, which truncates the otherwise $1 / r^{3}$ interaction, many bosonic oscillator systems are often naturally nearest neighbor. For example, the realization of such coupled oscillators is currently being explored in systems such as cavity arrays [23-25], nanomechanical oscillators [63,64], Josephson junctions [65-67], and optomechanical crystals [68].

Consider a chain of coupled harmonic oscillators with the Hamiltonian

$$
H_{B}=\sum_{i=1}^{N} \omega a_{i}^{\dagger} a_{i}+\sum_{i=1}^{N-1} \kappa\left(a_{i}^{\dagger} a_{i+1}+a_{i+1}^{\dagger} a_{i}\right) .
$$

As before, we begin by diagonalizing the Hamiltonian. Let us define $b_{k}=\frac{1}{A} \sum_{j} \sin \frac{j k \pi}{N+1} a_{j}$, with $A=\sqrt{(N+1) / 2}$ and $k=1, \ldots, N$, yielding $H=\sum_{k}\left(\omega+\epsilon_{k}\right) b_{k}^{\dagger} b_{k}$, where $\epsilon_{k}=$ $2 \kappa \cos \left(\frac{k \pi}{N+1}\right)$. The perturbative coupling of the two additional quantum registers at the ends of the oscillator chain is given by $H_{B}^{\prime}=g\left(a_{0}^{\dagger} a_{1}+a_{N}^{\dagger} a_{N+1}+\right.$ H.c. $)+\omega^{\prime}\left(a_{0}^{\dagger} a_{0}+a_{N+1}^{\dagger} a_{N+1}\right)$, where $g$ characterizes the register-oscillator-chain coupling strength and $\omega^{\prime}$ is the register frequency. Upon reexpressing $a_{1}$ and $a_{N}$ as a function of the eigenmodes $b_{k}$, we arrive at the full Hamiltonian

$$
\begin{aligned}
H_{B}+H_{B}^{\prime}= & \sum_{k=1}^{N} t_{k}\left[a_{0}^{\dagger} b_{k}+(-1)^{k-1} a_{N+1}^{\dagger} b_{k}+\text { H.c. }\right] \\
& +\omega^{\prime}\left(a_{0}^{\dagger} a_{0}+a_{N+1}^{\dagger} a_{N+1}\right)+\sum_{k=1}^{N}\left(\omega+\epsilon_{k}\right) b_{k}^{\dagger} b_{k},
\end{aligned}
$$

where we let $t_{k}=(g / A) \sin [k \pi /(N+1)]$. In analogy to Sec. II, we consider resonant tunneling through a particular mode $b_{z}$ by tuning $\omega^{\prime}=\omega+\epsilon_{z}$ and ensuring that $t_{z} \ll$ $\left|\epsilon_{z}-\epsilon_{z \pm 1}\right|$. The resulting effective Hamiltonian is $H_{\mathrm{eff}}^{B}=$ $\sqrt{2} t_{z}\left(\eta_{0}^{\dagger} b_{z}+b_{z}^{\dagger} \eta_{0}\right)$, where $\eta_{0}=1 / \sqrt{2}\left(a_{0}+a_{N+1}\right)$. To demonstrate state transfer, we introduce operators $\xi_{ \pm}=1 / \sqrt{2}\left(\eta_{0} \pm\right.$ $\left.b_{z}\right)$, yielding

$$
H_{\mathrm{eff}}^{B}=\sqrt{2} t_{z}\left(\xi_{+}^{\dagger} \xi_{+}+\xi_{-}^{\dagger} \xi_{-}\right) .
$$

Let us now consider unitary evolution under $H_{\text {eff }}^{B}$ for a time $\tau_{B}=\pi / \sqrt{2} t_{z}$, wherein $U_{\text {eff }}^{B}=e^{-i H_{\text {eff }}^{B} \tau_{B}}=(-1)^{\xi_{+}^{\dagger} \xi_{+}}(-1)^{\xi_{-}^{\dagger} \xi_{-}}$, so that $\left(U_{\mathrm{eff}}^{B}\right)^{\dagger} \xi_{ \pm}\left(U_{\mathrm{eff}}^{B}\right)=-\xi_{ \pm}$. Returning to the original basis and evaluating the time evolution of $a_{0}$ and $a_{N+1}$ yields

$$
\begin{aligned}
a_{0}(\tau) \rightarrow\left(U_{\text {eff }}^{B}\right)^{\dagger} a_{0}\left(U_{\text {eff }}^{B}\right) & =-a_{N+1}, \\
a_{N+1}(\tau) \rightarrow\left(U_{\text {eff }}^{B}\right)^{\dagger} a_{N+1}\left(U_{\text {eff }}^{B}\right) & =-a_{0},
\end{aligned}
$$

demonstrating a SWAP gate between the oscillator registers at the ends of the chain. As before, this state transfer is achieved independent of the state of the intermediate chain. Moreover, there exists no additional entanglement between the registers and the intermediary oscillators; this is a direct consequence of the bosonic nature of the modes, which, unlike their Wignerfermionic counterparts in Sec. II, carry no strings.

One crucial difference with the spin chain case is that the occupation of the bosonic eigenmodes is not limited to 0 or 1 . In a highly excited system, this induces a bosonic enhancement of off-resonant errors and will limit the achievable state transfer fidelity as a function of temperature. In particular, the state-transfer unitary evolution gives $a_{N+1}(\tau)=M_{N+1,0} a_{0}+$ $\sqrt{\epsilon} a_{\epsilon}$, where $\epsilon=1-\left|M_{N+1,0}\right|^{2} \propto g^{2}$ is a small error and $a_{\epsilon}$ is a normalized linear combination of the $a_{i}$ modes $(i=$ $1, \ldots, N+1)$. The total number of excitations in mode $N+1$ after the state transfer is $\left\langle n_{N+1}(\tau)\right\rangle=(1-\epsilon)\left\langle n_{0}\right\rangle+\epsilon\left\langle n_{\epsilon}\right\rangle$, where $n_{i}=a_{i}^{\dagger} a_{i}$. Therefore, if the chain is thermally occupied with $\left\langle n_{\epsilon}\right\rangle \approx k T / \omega>1$, the coupling strength $g$ must be reduced to $g \sqrt{\omega / k T}$ in order to keep errors comparable with the zero-temperature bosonic case. In realistic experimental systems, this implies an interplay between temperature, which sets the Bose enhancement of off-resonant errors and decoherence rates, which limit the minimal speed of state transfer.

\section{DISORDER AND DECOHERENCE}

Eigenmode-mediated state transfer naturally finds use in a variety of quantum computing architectures where data buses are required to connect high-fidelity remote registers $[3,4,47]$. Within such architectures, it is crucial to consider an interplay between naturally occurring disorder and finite decoherence rates. While disorder in one-dimensional (1D) systems generically localizes all eigenmodes [48,55,69,70], leading to an exponentially long state-transfer time, in finitesize systems with weak disorder, the localization lengths can be large relative to the inter-register separation. In these cases, one must still reduce the register-chain coupling strength $g$ to compensate for disorder effects, but so long as the register decay time is sufficiently long, it remains possible to achieve high-fidelity QST. In this section we will discuss the impact of 
coupling-strength disorder on spin chains and will analyze the optimization of $g$ as a function of disorder strength and qubit depolarization time.

In particular, we will consider two sources of error: (i) off-resonant coupling to alternate eigenmodes (which becomes enhanced as disorder increases) and (ii) a finite register depolarization time $T_{1}$,

$$
\epsilon=\sum_{k \neq z}\left(g_{L}^{2} \frac{\left|\psi_{k, L}\right|^{2}}{\Delta_{k}^{2}}+g_{R}^{2} \frac{\left|\psi_{k, R}\right|^{2}}{\Delta_{k}^{2}}\right)+N \frac{t}{T_{1}},
$$

where $g_{L(R)}$ is left (right) register-chain coupling, $\psi_{k, L(R)}$ is the eigenmode amplitude at the left (right) register, $\Delta_{k}$ is the energy difference from mode $z$ to mode $k, N$ is the chain length, $t$ is the state transfer time, and $T_{1}$ is the depolarization time of the register. The additional factor of $N$ in the final term results from the entanglement discussed in Sec II; indeed, since each register is $\mathrm{CP}$ entangled with all intermediate spins, any spin flip of the intermediate chain immediately dephases the quantum information.

To ensure that the tunneling rates at each end of the intermediate chain are equivalent, we envision tuning $g_{L}$ and $g_{R}$ independently such that $t_{z}=g_{L}\left|\psi_{z, L}\right|=g_{R}\left|\psi_{z, R}\right|$. Plugging in for the state-transfer time $t=\pi / \sqrt{2} t_{z}$ yields

$\epsilon=\sum_{k \neq z} g_{L}^{2}\left(\frac{\left|\psi_{k, L}\right|^{2}}{\Delta_{k}^{2}}+\frac{\left|\psi_{z, L}\right|^{2}}{\left|\psi_{z, R}\right|^{2}} \frac{\left|\psi_{k, R}\right|^{2}}{\Delta_{k}^{2}}\right)+\frac{N \pi}{\sqrt{2} T_{1} g_{L}\left|\psi_{z, L}\right|}$,

which enables us to derive the optimal coupling strength

$$
g_{L}=\sqrt[3]{\frac{N \pi}{2 \sqrt{2} T_{1}\left|\psi_{z, L}\right|}\left(\sum_{k \neq z} \frac{\left|\psi_{k, L}\right|^{2}}{\Delta_{k}^{2}}+\frac{\left|\psi_{z, L}\right|^{2}}{\left|\psi_{z, R}\right|^{2}} \frac{\left|\psi_{k, R}\right|^{2}}{\Delta_{k}^{2}}\right)^{-1}} .
$$

\section{A. Disorder numerics for a specific NV-based architecture}

We now consider an example implementation of eigenmode-mediated state transfer in the context of a quantum computing architecture based upon nitrogen-vacancy registers in diamond [49-51]. Each fully controllable NV register consists of a coupled electronic and nuclear spin. The nuclear spin, with extremely long multisecond room-temperature coherence times, is often thought of as the memory qubit [8], while the electronic spin, which can be optically initialized and read out, mediates interactions with other NVs $[3,47]$. Our analysis of disorder effects will be based upon the specific architecture proposed in Ref. [3]; there NV registers are connected by a dark-spin-chain data bus composed of spin- $1 / 2$ electronic spins associated with nitrogen impurities. One of the crucial advantages of utilizing spin chains to connect remote NVs is that this enables optical addressing of individual registers in parallel, a necessary requirement for the implementation of many error-correcting codes.

We consider realistic experimental parameters, with an average spin spacing of about $10 \mathrm{~nm}$, corresponding to a dipole coupling strength $\approx 50 \mathrm{kHz}$. At room-temperature, NV centers are characterized by $T_{1} \sim 10 \mathrm{~ms}$ [8], owing to an Orbach spin-lattice relaxation process; the exponential dependence of the Orbach process on temperature suggests that slight cooling can significantly extend $T_{1}$, with many seconds already demonstrated at liquid nitrogen temperatures [3,71]. We now perform disorder-averaged numerics for two separate chain lengths: (i) subwavelength addressable $(N=$ 11) and (ii) optical-wavelength addressable $(N=51)$ [72]. We characterize the amount of disorder by the standard deviation associated with imperfect spin positioning; in the case of NVs, the origin of this imperfection is straggle during the ion-implantation process [73,74]. We average over 1000 disorder realizations and calculate the fidelity $1-\epsilon$ according to Eq. (28); in particular, for each realization, we calculate the error for each eigenmode of the spin chain and choose the maximum achievable fidelity. As shown in Fig. 3(a), high-fidelity quantum gates can be achieved for few-nanometer straggle provided that the NV depolarization time is $\sim 200 \mathrm{~ms}$; similarly, for the longer chain case [Fig. 3(b)] with $N=51$, high-fidelity gates are also possible, but require significantly longer $T_{1}$ of a few seconds.

Next we analyze the participation ratio (PR) $[55,70]$

$$
N_{\mathrm{PR}}=\frac{1}{\sum_{i=1}^{N}\left|\psi_{i}\right|^{4}},
$$

which provides a characterization of the number of sites that participate in a given eigenmode; modes are typically said to be extended if $N_{\mathrm{PR}} \sim O(N)$ and localized if $N_{P R} \ll N$. In the case where the participation ratio is much shorter than the overall chain length, it becomes extremely difficult to perform quantum state transfer within the coherence window set by the quantum register. This allows us to quantitatively determine the regime over which high-fidelity state transfer can be achieved despite the interplay between disorder and decoherence. As shown in the histograms of Fig. 3(c), for increasing disorder, $N_{\mathrm{PR}}$ drops sharply as a function of $\sigma_{\kappa}$. By $\sigma_{\kappa} \approx 0.5 \kappa$, on average, all eigenmodes exhibit a state-transfer fidelity less than $2 / 3$ even for extremely long $T_{1} \sim 5 \mathrm{~s}$.

\section{STRONG REGISTER COUPLING}

The eigenmode-mediated QST discussed above operates in the weak-coupling regime $g \psi \ll \kappa / N$. Numerical simulations reveal that by optimally tuning $g=g_{M}(N) \sim \kappa$, high-fidelity QST can also be achieved (see Fig. 5). This strong-coupling regime enables faster state transfer and has been analyzed in several recent studies $[18,39,54,55,75]$. Here our contribution is to show that this strong-coupling regime still enables high-fidelity state transfer in the case of unpolarized channels. We demonstrate this by performing numerical simulations and deriving analytic channel fidelities valid for an arbitrary coupling pattern (analogous to Sec. III). We emphasize that our results are consistent with and motivated by those in Refs. [18,39,54,55,75].

To provide intuition for this strong-coupling regime [18, $39,54,55]$, we will begin by considering the engineered spin chain described in Ref. [31], where we have $N+2$ spin-1/2 atoms with nearest-neighbor $X X$ interactions. The intrachain coupling is nonuniform and is given by, 

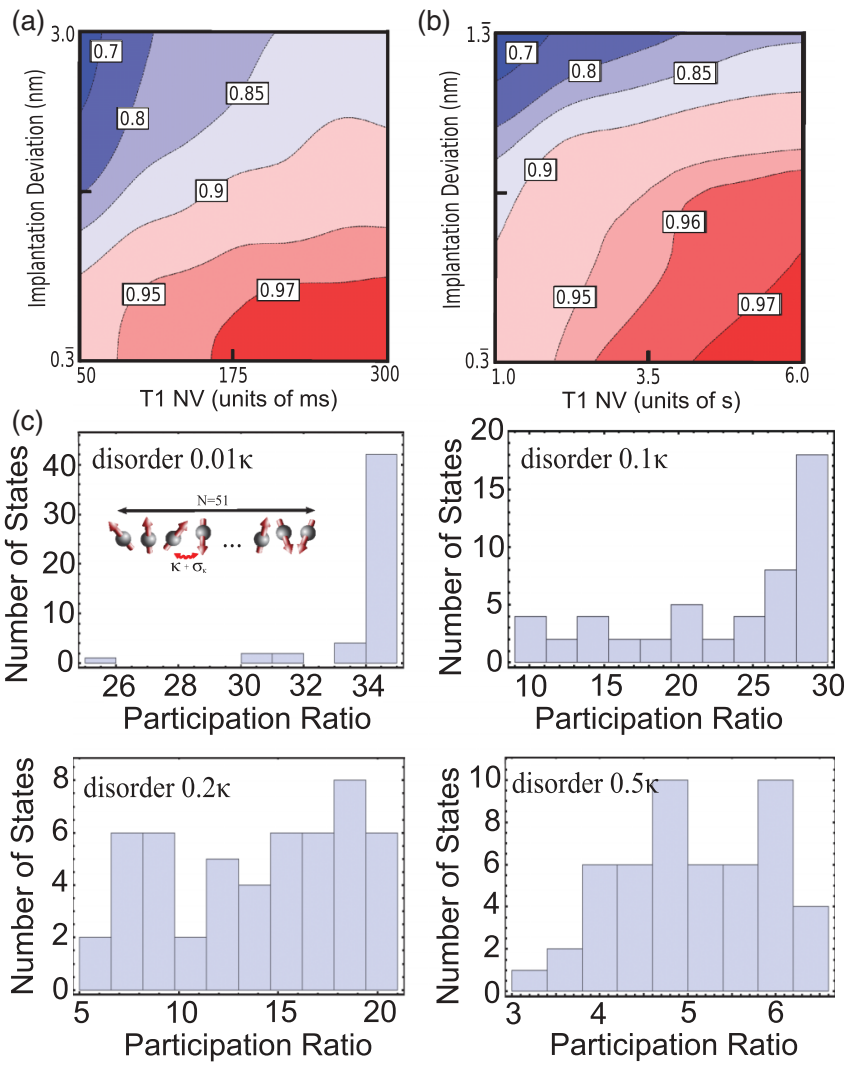

FIG. 3. (Color online) (a) Contour plots for $N=11$ characterizing the average achievable fidelity as a function of the NV depolarization time $T_{1}$ and the coupling strength disorder induced by imperfect implantation. Numerics utilize an average intrachain spin spacing of $d=10 \mathrm{~nm}$ corresponding to a $\kappa=50 \mathrm{kHz}$ dipoledipole interaction strength. This intrachain spacing is assumed to be Gaussian distributed and the implantation deviation represents the standard deviation $\sigma_{d}$. For each $\sigma_{d}, 1000$ realizations were averaged to obtain the plotted fidelity and a smooth contour plot is generated via a third-order spline interpolation. The register-chain coupling strengths $g_{L}$ and $g_{R}$ [Eq. (29)] are assumed to be fully tunable via control of the three-level NV ground-state manifold [3]. (b) Analogous contour plots for $N=51$. In this case, the NV registers are separated by an order optical wavelength enabling individual laser manipulation without the need for subwavelength techniques. (c) Participation ratio for eigenmodes $(N=51)$. Each eigenmode is indexed by its PR and the number of states within a certain PR bin is shown. For each disorder (which are represented as fractions of the bare coupling strength $\kappa=50 \mathrm{kHz}$ ), 1000 realizations are averaged.

$J_{i}=\frac{1}{2} \sqrt{(i+1)(N+1-i)}$, yielding a Hamiltonian

$$
H=\sum_{i=0}^{N} J_{i}\left(\sigma_{i}^{+} \sigma_{i+1}^{-}+\text {H.c. }\right)+\sum_{i=0}^{N+1} \frac{h}{2} \sigma_{i}^{z},
$$

where $h$ is a uniform background magnetic field. Upon employing the Jordan-Wigner transformation, we once again return to a simple tight-binding form, with $H=\sum_{i j} K_{i j} c_{i}^{\dagger} c_{j}$, where $K_{i j}=J_{i} \delta_{j, i+1}+J_{j} \delta_{i, j+1}+h \delta_{i, j}$ up to a constant. Diagonalizing reveals $H=\sum_{k=0}^{N+1} \omega_{k} f_{k}^{\dagger} f_{k}$ with a linear spectrum given by $\omega_{k}=k+h-\frac{N+1}{2}$.
As described in Sec. III, the system's evolution is governed by $c_{i}(t)=\sum_{j} M_{i j}(t) c_{i}(0)$. Upon setting $h=\frac{N+1}{2}$, one finds that at time $t=2 \pi, M(2 \pi)=\mathbb{I}$ and therefore $c_{i}(2 \pi)=c_{i}(0)$, returning the system to its initial state. As the coupling pattern $\left\{J_{i}\right\}$ harbors mirror symmetry with $J_{i}=J_{N-i}$, the orthogonal transformation $\psi$ that diagonalizes $H$ can also be chosen mirror symmetric, $\psi_{i k}=(-1)^{N+1+k} \psi_{N+1-i, k}$. Setting $h=\frac{3}{2}(N+1)$ and $t=\pi$ yields

$$
M_{i j}=\sum_{k} \psi_{N+1-i, k} \psi_{j k}=\delta_{N+1-i, j}
$$

To demonstrate state transfer, let us recall the analytic singleswap fidelity given by Eq. (20). For the moment, let us assume that the spins $\{0,1, \ldots, N\}$ are all polarized so that $\operatorname{Tr}\left[\rho_{\mathrm{ch}}^{\mathrm{SS}} P\right]=$ 1. Combined with Eq. (32), which ensures $M_{0, N+1}=1$, we find $F_{\mathrm{SS}}=1$, enabling perfect QST. We note that in lieu of applying a uniform magnetic field $h=\frac{3}{2}(N+1)$, one can also just apply a simple PHASE gate $U_{P}=\left(\begin{array}{cc}1 & 0 \\ 0 & (-i)^{N+1}\end{array}\right)$ on spin 0 following transfer.

Turning now to the case of an unpolarized spin chain, we again employ the two-qubit encoding previously described. In this case, one will need to apply the PHASE gate $U_{P}^{2}=$ $\left(\begin{array}{cc}1 & 0 \\ 0 & (-1)^{N+1}\end{array}\right)$ to the logical qubit after state transfer.

The state-transfer fidelities for these two strong-coupling methods are given analogously by

$$
\begin{gathered}
F_{\mathrm{SS}}=\frac{1}{2}+\frac{1}{6}\left(2\left|M_{0, N+1}\right|+\left|M_{0, N+1}\right|^{2}\right) \\
F_{\mathrm{enc}}=\frac{1}{2}+\frac{1}{6}\left(2\left|M_{0, N+1}\right|^{2}\left|M_{0, N+1}^{2}-M_{0,0} M_{N+1, N+1}\right|\right. \\
\left.+\left|M_{0, N+1}\right|^{2}+\left|\sum_{i} M_{N+1, i} M_{i, 0}\right|^{2}\right)
\end{gathered}
$$

While these expressions are valid for an arbitrary coupling pattern (so long as the resultant fermionic Hamiltonian is quadratic), to ensure high-fidelity QST we require $\left|M_{0, N+1}\right| \approx$ 1. As depicted in Eq. (32), satisfying this constraint is intimately related to the linear spectrum resulting from the choice of $J_{i}=\frac{1}{2} \sqrt{(i+1)(N+1-i)}$.

Let us now consider the strong-coupling regime $(g \sim \kappa)$ where $\quad J_{0}=J_{N}=g \quad$ and $\quad J_{1}=J_{2}=\cdots=J_{N-1}=\kappa$. Surprisingly, tuning only $g / \kappa$ enables one to obtain a quasilinear spectrum [18]; such a spectrum will then ensure that $\left|M_{0, N+1}\right| \approx 1$, as desired. Of course, for $N=2,3$, $J_{i}=\frac{1}{2} \sqrt{(i+1)(N+1-i)}$ can be satisfied exactly. Although for $N>3$, an exactly linear spectrum cannot be obtained, it is possible to optimally tune $g=g_{M}(N)$ so that $\omega_{k}$ looks nearly identical to the previous linear spectrum $k-\frac{N+1}{2}$ $(h=0)$, as shown in Fig. 4. In particular, by optimizing $F_{\text {enc }}^{2}$, we obtain $g_{M} \sim N^{-1 / 6}$, with a transfer time $\tau \sim N$ (Fig. 5), consistent with Ref. [18]. Interestingly, for extremely long chains with $N>100$, the optimal coupling strength is not necessarily the one that best linearizes the dispersion, implying a more complex interplay between state transfer and non-linearities [54,75]. 


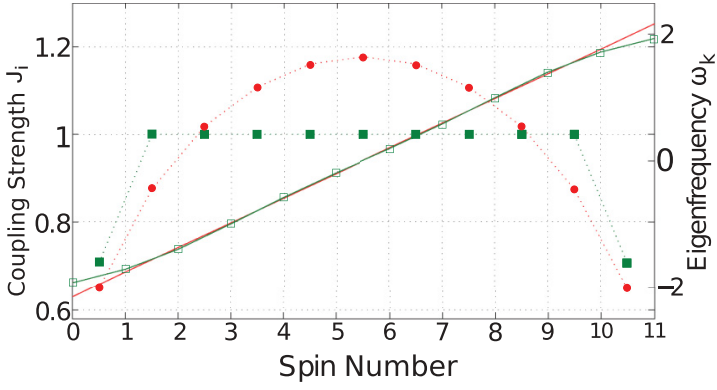

FIG. 4. (Color online) Coupling pattern $\left\{J_{i}\right\}$ between spins for two different cases: (i) engineered couplings (circles) as in Ref. [31] and (ii) the strong-coupling regime (squares). The left $y$ axis characterizes the coupling strength for each case and is associated with solid symbols; the couplings are plotted between spin numbers (e.g., $J_{0}$ is plotted between spin numbers 0 and 1). The right $y$ axis characterizes the fermionic spectrum (in this case, the $x$ axis is simply an index) and is associated with the open symbols. The open red circles depict the exactly linear spectrum of the engineered chain, while the open green squares depict the quasilinear spectrum of the strong-coupling case with uniform interchain couplings $\kappa=1$ and optimized $g \approx 0.7$.

\section{LONG-RANGE INTERACTIONS}

Finally, we now consider the effect of longer-range interactions. The majority of proposals for spin-chain state transfer focus on approximate nearest-neighbor models; however, the microscopic magnetic dipolar interaction is naturally long range and decays as $1 / r^{3}$, inducing an important infidelity in quantum state transfer. The origin of this infidelity becomes especially evident as we examine the Jordan-Wigner fermionization of the spin chain. Each Wigner fermion carries a string of the form $\exp \left(-i \pi \sum_{j=1}^{i-1} \sigma_{j}^{+} \sigma_{j}^{-}\right)$. In the nearest-neighbor case, all such strings cancel pairwise, leaving a simple quadratic model. However, with longer-range interactions, uncanceled strings remain and generically introduce perturbative quartic
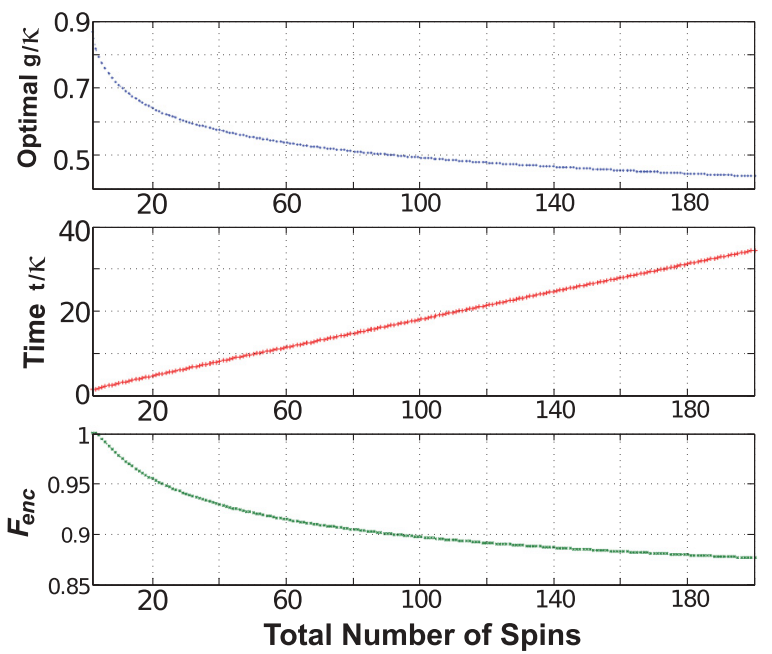

FIG. 5. (Color online) Strong-coupling regime: By tuning $g / \kappa \sim$ $N^{-1 / 6}$, we obtain high-fidelity QST utilizing an unpolarized chain with two-qubit encoding (paired protocol). The transfer time scales linearly with $N$ (Lieb-Robinson bound) [76] and high fidelities greater than $90 \%$ can be maintained for chain lengths up to $N=100$.

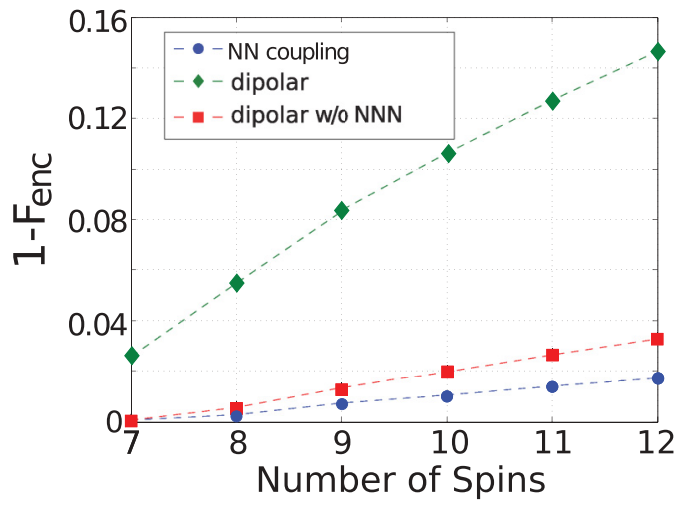

FIG. 6. (Color online) Infidelity of QST for a strongly coupled chain with long-range interactions ( $1 / T_{1}$ is assumed to be negligible). Encoded state-transfer (paired-protocol) fidelities are shown for dipolar (diamonds), NNN-canceled-dipolar (squares), and NN interaction (circles) models.

terms into the Hamiltonian. These quartic terms imply that the model, unlike the transverse field Ising model, is no longer diagonalizable in terms of free fermions. In the previous free fermion case, the energy of each eigenmode is independent of the occupation of all other eigenmodes; this enables state transfer even when the spin temperature of the chain is effectively infinite. By contrast, the quartic terms associated with the long-range dipolar coupling introduce interactions between fermionic eigenmodes; the energy fluctuations of each eigenmode, caused by changing occupations of other modes, naturally dephase quantum information, limiting the operational spin temperature of the chain.

Certain proposals have suggested the possibility of using dynamical decoupling to effectively cancel next-to-neareastneighbor (NNN) interactions [3], but the complete canceling of all long-range interactions requires a level of quantum control that is currently beyond the realm of experimental accessibility. Since any long-range $X X$ coupling destroys the quadratic nature of the fermionic Hamiltonian, an analytic solution for state-transfer fidelities in the presence of full dipolar interactions is not available. Thus we perform exact diagonalization for chains of length up to $N=12$ (total number of spins), as shown in Fig. 6. We obtain the encoded state-transfer fidelities for dipolar, NNN-canceled-dipolar, and NN interaction models. Remarkably, even with full dipolar interactions, fidelities of $\sim 90 \%$ can be obtained for a total of $N=10$ spins; in the case where NNN interactions are dynamically decoupled, the fidelities can be further improved to $\sim 98 \%$ at similar lengths.

\section{QUANTUM MIRROR ARCHITECTURE}

In this section we present an alternate quantum computing architecture based upon pulsed quantum mirrors [43,44]. By contrast to eigenmode-mediated state transfer, remote quantum logic will be achieved by global rotations and NN Ising interactions. To remain consistent, we choose to discuss the advantages and disadvantages of such an architecture within the context of NV registers. In particular, analogous to Sec. V, we consider NV registers connected by spin-1/2 chains of implanted nitrogen impurities. 
Let us begin with a detailed discussion of the mixed spin system composed of NV centers and nitrogen impurities [3]. The full Hamiltonian of a single nitrogen impurity is

$H_{N}=-\gamma_{e} \vec{B} \cdot \vec{S}-\gamma_{N} \vec{B} \cdot \vec{I}+A_{\|} S^{z} I^{z}+A_{\perp}\left(S^{x} I^{x}+S^{y} I^{y}\right)$,

where $\vec{S}$ is the spin- $1 / 2$ electronic spin operator, $\vec{I}$ is the nuclear spin operator, and $A_{\|}=-159.7 \mathrm{MHz}$ and $A_{\perp}=-113.8 \mathrm{MHz}$ are the hyperfine constants associated with the Jahn-Teller axis.

We envision the application of a magnetic-field and field gradient, which, within a secular approximation, reduces the Hamiltonian of a nearest-neighbor nitrogen-impurity chain to Ising form [3]

$$
H_{N}=\kappa \sum_{i=1}^{N-1} S_{i}^{z} S_{i+1}^{z}+\sum_{i=1}^{N}\left(\omega_{0}+\delta_{i}\right) S_{i}^{z}
$$

where $\kappa$ is the relevant component of the dipole tensor, $\omega_{0}$ captures the electronic Zeeman energy, and $\delta_{i}$ characterizes the hyperfine term, which is nuclear-spin dependent, for each impurity. Taking into account the magnetic dipole coupling between the electronic spin of the NV register and the surrounding nitrogen impurities allows us to consider the mixed spin system

$$
H_{\mathrm{eff}}=\sum_{i=1}^{a-1} \kappa S_{i}^{z} S_{i+1}^{z}+J S_{\mathrm{NV}}^{z}\left(S_{a}^{z}+S_{b}^{z}\right)+\sum_{i=b}^{N-1} \kappa S_{i}^{z} S_{i+1}^{z},
$$

where $J$ is the strength of register-impurity interaction, the Zeeman term in Eq. (36) is assumed to be echoed out, and superscripts $a$ and $b$ represent the pair of nearest-neighbor impurities next to a given register (assuming for simplicity a $1 \mathrm{D}$ geometry as shown in Fig. 7). The selective individual addressing of the NV registers is accomplished via a combination of optical beams and microwave driving; this enables an isolation of the coupling between the NV register and the two neighboring impurities. In particular, it is possible to perform unitary evolution of the form

$$
\begin{aligned}
U_{\mathrm{eff}} & =e^{-i H_{\mathrm{eff}} T^{\prime} / 2} S_{\mathrm{NV}}^{x} e^{-i H_{\mathrm{eff}} T^{\prime} / 2} S_{\mathrm{NV}}^{x} \\
& =\exp \left(-i \kappa \sum S_{i}^{z} S_{i+1}^{z} T^{\prime}\right)
\end{aligned}
$$

and hence

$U_{\text {local }}=e^{-i H_{\text {eff }} T} \exp \left(-i \kappa \sum S_{i}^{z} S_{i+1}^{z} T^{\prime}\right)=e^{-i J S_{\mathrm{NV}}^{z}\left(S_{a}^{z}+S_{b}^{z}\right) T}$

by choosing $\kappa\left(T+T^{\prime}\right)=2 \pi m$ for integer $m$. We note that this condition implies that the fidelity of $U_{\text {local }}$ is extremely sensitive to both coupling-strength disorder and the general long-range nature of the dipolar interaction.

\section{A. Globally controlled mirror swap}

Considering only global addressing of the nitrogen spin chain and unitary evolution as described above, we demonstrate a universal set of operations between remote NV registers. Coherent register coupling is achieved by means of global pulses that mirror the quantum state of the impurity chain [43]; the pulses take the form of Hadamard gates and

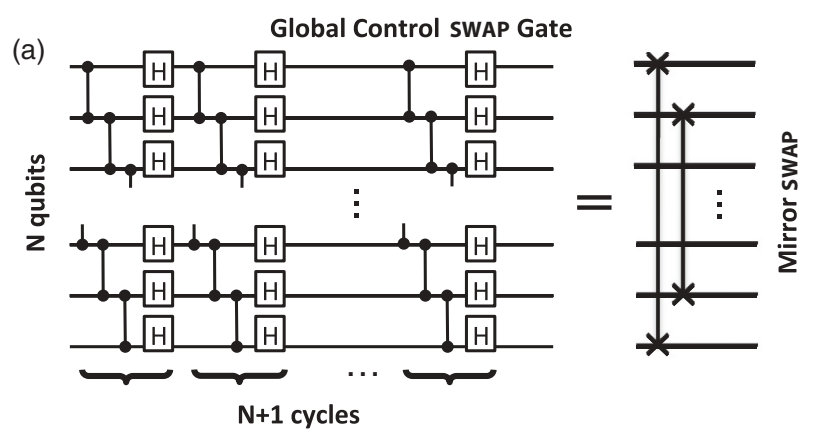

(b)
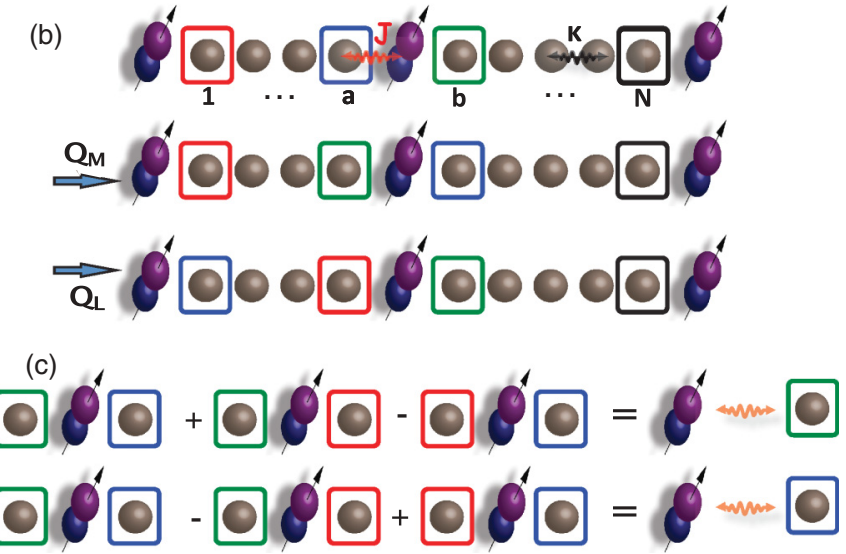

FIG. 7. (Color online) (a) In a qubit chain of length $N$, a mirror SWAP operation is defined as the pairwise swap between the $(1, N),(2, N-1), \ldots$ qubits. This can be achieved via global control in the form of single-qubit gates (Hadamards) and controlled-PHASE gates. Regardless of the initial state, a mirror swap occurs after a $N+1$ cycles of $Q=\tilde{H} \tilde{C P}$ [43], where $\tilde{H}$ represents a global Hadamard operation and $\tilde{C P}$ denotes a global controlled-PHASE operation. (b) Coupling the central NV register to the NV on the left (right) requires the ability to perform a swap directed to a neighboring nitrogen spin (gray). These directed SWAP operations are made possible by using combinations of the mirror sequences $Q_{M}$ (swaps a pair of impurities directly surrounding a NV) and $Q_{L}$ (mirror swaps an individual impurity chain). (c) Utilizing a fast echo pulse on the NV register (in combination with $Q_{M}$ and $Q_{L}$ ) allows one to generate selective interactions between the $\mathrm{NV}$ and any outlined nitrogen.

controlled-PHASE gates, which can be generated by evolution under an Ising Hamiltonian. In an impurity spin chain of length $N$, the global pulses swap the state of the first and $N$ th spin, the state of the second and $(N-1)$ st spin, etc., as shown in Fig. 7(a). The total mirror swap results from $N+1$ cycles of Hadamard and controlled-PHASE gates on all impurities $Q_{n+1}=\left(\prod H_{i} \prod \mathrm{CP}_{i}\right)^{n+1}$. This globally controlled impurity mirror will ultimately enable the directed and coherent interaction between remote $\mathrm{NV}$ registers.

Let us now consider a specific NV register, separated from neighboring registers by impurity spin chains on both sides, as shown in Fig. 7(b). Since the Ising Hamiltonian generates a controlled-PHASE gate, it is possible to achieve a mirror swap between any set of qubits connected by Ising interactions. In particular, the impurity Ising interaction allows for a mirror operation within any impurity chain, while the Ising interaction corresponding to $U_{\text {local }}$ allows for a three-qubit mirror centered 


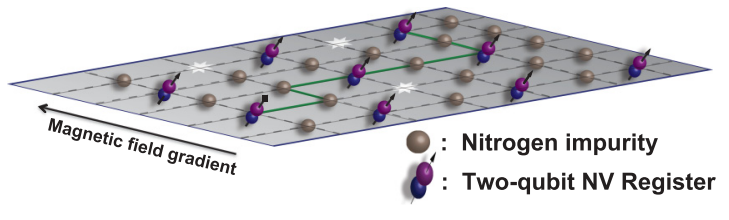

FIG. 8. (Color online) Schematic diagram of the 2D computational lattice showing limitations imposed by missing nitrogen implantations (stars) and imperfect nitrogen-to-NV conversions. Coherent coupling of distant NV registers in a faulty $2 \mathrm{D}$ array can be achieved via global pulsed control of a spin-chain quantum data bus. A combination of optical beams and a magnetic-field gradient allows for individual control of NV registers; combined with global single-qubit operations on nitrogen impurities in any given row (orthogonal to the field gradient) [3], directed SWAP operations (e.g., dark green path) can be achieved, which allow for quantum information transfer along arbitrary paths. This field gradient enables a SWAP gate to be performed between two NV registers in adjacent rows, which occupy the same column. Moreover, it in fact also enables any pair of rows to be swapped provided the intrarow interactions refocus.

around any NV register. This local unitary enables an operation $Q_{M}$ that swaps the state of the nitrogen neighbors of the central NV register as shown in Fig. 7(b).

To couple the central NV register to a specific side register, it will be necessary to break the left-right symmetry of the Ising interaction; this is achieved by exploiting the length asymmetry between nitrogen chains to the left and right of the NV register. Indeed, it is often possible to refocus the mirror operation in one impurity chain while causing the edge impurity pair to swap in the other chain; we will denote this operation as $Q_{L}$, as shown in Fig. 7(b). Combinations of $Q_{M}$ and $Q_{L}$ successfully manipulate and permute the impurities such that the nearest neighbors of the central NV register can be any pair of the three impurities (blue, red, and green), as depicted in Fig. 7(c). In combination with local rotations of the central register, this enables the application of directed unitary evolution, e.g., $U_{\text {directed }}=e^{-i J S_{\mathrm{NV}}^{z} S_{N_{b}}^{z} T}$, allowing for the NV register to selectively couple to either side. This enables an interaction between any pair of neighboring NV registers effectively mediated by a single nitrogen impurity

$$
H_{\mathrm{med}}=J\left(S_{\mathrm{NV}_{1}}^{z}+S_{\mathrm{NV}_{2}}^{z}\right) S_{N_{b}}^{z},
$$

where $\mathrm{NV}_{1}$ and $\mathrm{NV}_{2}$ denote the neighboring registers to be coupled and $N_{b}$ represents the mediating impurity. The form of this Ising interaction implies that an application of $Q_{M}$ on this effective three-qubit system will swap the quantum information of the two electronic spins of the remote NV registers. Since each NV center harbors a nuclear-spin qubit in addition to its electronic spin [49], the first protocol described in Sec. II enables universal logic between remote registers.

Having achieved the ability to coherently couple distant NV registers within a row, assisted by nitrogen impurities, we now turn to the coupling between adjacent rows in a twodimensional lattice (Fig. 8). The simplest approach involves applying a magnetic-field gradient along the columns. This would enable a SWAP gate to be performed between two NV registers in adjacent rows, which occupy the same column, provided all other interactions are echoed out. The limited

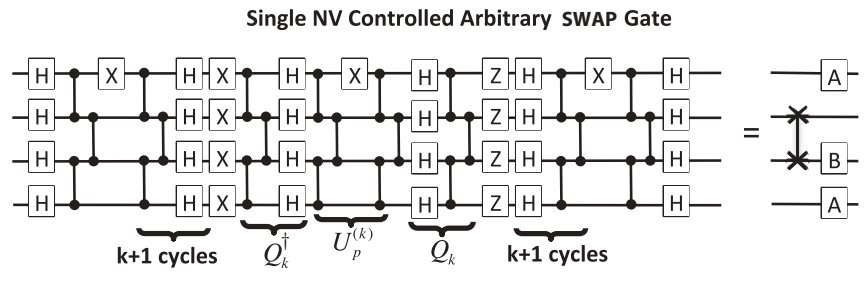

FIG. 9. Individual control of any given NV register (row 1) enables a SWAP operation between any two neighboring qubits along the same row. We illustrate the specific example of a four-qubit chain. The depicted gate sequence achieves a SWAP gate (up to individual qubit rotations $A$ and $B$ ) between the second and third qubit by only applying a local gate $X$ on the first qubit (NV register) and global operations elsewhere.

occurrence of vertically adjacent $\mathrm{NVs}$ is a significant source of overhead; however, this limitation can be overcome if we achieve the ability to swap any pair of nearest-neighbor qubits in the two-dimensional array, essentially allowing for the construction of arbitrary paths (Fig. 8). Moreover, the ability to swap along arbitrary paths also provides an elegant solution to the experimental limitation imposed by implantation holes, where a nitrogen impurity may be missing from the ideal 2D lattice. Finally, it also enables the use of nominally dark nitrogen impurities as computational resources, thereby significantly increasing the number of effectively usable qubits.

While arbitrary individual control of impurities would trivially enable such a scheme, realistic constraints limit us to individual control of NV registers and only global control of the impurity chains. Thus it is necessary to utilize the permutation operation inherent to individual cycles $\left(\prod H_{i} \prod \mathrm{CP}_{i}\right)^{n+1}$ of the mirror operation. These gate cycles correspond to an effective propagation of local gates via a relabeling of qubits within a given chain. In the simplest scenario, it is possible to apply a SWAP gate between the second and third qubit by only utilizing local rotations on the first qubit and global operations elsewhere, as shown in Fig. 9. The fundamental operation to be propagated is $U_{p}=\tilde{\mathrm{CP}} X_{1} \tilde{\mathrm{CP}}$, where $X_{1}$ is an $x$ rotation (by $\pi$ ) on the first qubit and CP represents a global controlled-PHASE gate; propagation takes the form of conjugation by mirror cycles where $Q_{k}=(\tilde{H} \tilde{\mathrm{CP}})^{k}$ and $U_{p}^{(k)}=Q_{k}^{\dagger} U_{p} Q_{k}$. To apply a SWAP operation on the $n$ and $n+1$ qubits, we let $k=n-1$ and apply

$$
U_{\mathrm{SWAP}}=\tilde{H} U_{p}^{(k)} \tilde{H} \tilde{X} U_{p}^{(k)} \tilde{Z} \tilde{H} U_{p}^{(k)} \tilde{H},
$$

where $\tilde{X}$ is a global $x$ rotation and $\tilde{Z}$ is a global $z$ rotation (by $\pi$ ). This protocol requires the ability to produce a boundary at the location of the first qubit and allows for swaps between arbitrary spins in a given row; moving quantum information between rows can be achieved provided intrarow interactions refocus (e.g., if vertical and horizontal nearest-neighbor distances differ).

\section{CONCLUSION}

In summary, we have extended the analysis of eigenmodemediated state transfer to a variety of imperfections ranging from disorder-driven localization to uncompensated longrange interactions. By calculating the analytic channel fidelity 
associated with eigenmode-mediated state transfer, we clarify the effects of entanglement arising from the protocol and illustrate the method in which the two-qubit encoding overcomes this challenge. We analyze our protocol in the context of proposed solid-state quantum computing architectures; numerical simulations with realistic experimental parameters reveal that QST errors can be kept below certain surface-code error-correcting thresholds. Furthermore, we have generalized our protocol to the case of bosonic oscillator systems. This approach may enable the routing of a ground-state-cooled mode through a relatively hot intermediate oscillator chain, thereby significantly reducing the resources associated with system-wide cooling.

Moreover, our work may also provide insight into generalized infinite-temperature state transfer. In particular, by introducing a time-dependent control of the register-chain coupling, one may be able to compensate for off-resonant errors. This approach finds analogy to the continuum wavepacket limit, where dispersion limits transfer fidelities; in this case, preshaping of the packet can overcome nonlinearities of the dispersion.

Finally, we describe an alternate architecture based upon global control pulses that also enables remote quantum logic; in particular, we demonstrate that even intermediate chain spins can be used as registers, despite the fact that they are unable to be individually addressed. This may provide the blueprint for a novel quantum computing architecture that utilizes dark spins as quantum memory resources.

\section{ACKNOWLEDGMENTS}

We gratefully acknowledge the insights of and conversations with Pierre Meystre, Alex Zhai, Sidd Viswanathan, Peter Komar, and Hendrik Weimer. This work was supported by the NSF, DOE, CUA, DARPA, AFOSR MURI, NIST, Lee A. DuBridge Foundation, Sherman Fairchild Foundation, IQIM, and the Gordon and Betty Moore Foundation. Z.-X.G. and L.-M.D. were supported by the ARO and the AFOSR MURI program, the IARPA MUSIQC program, the DARPA OLE program, and the NBRPC (973 Program Grants No. 2011CBA00300 and No. 2011CBA00302).

\section{APPENDIX A: PERTURBATIVE CALCULATION OF CHANNEL FIDELTY}

As an extension of the analytic fidelity derivations presented in Sec. III, here we provide a closed-form expression for certain relevant matrix elements. We will work perturbatively $(g \ll$ $\kappa / \sqrt{N}$ ) and will begin with the case of odd chain length. Let us consider computing $1-|\langle 0|M| N+1\rangle|^{2}$ for $z=(N+1) / 2$. Recall that $K$ is the $(N+2) \times(N+2)$ coupling matrix of the full $X X$ Hamiltonian. We can characterize it with basis $|j\rangle$ $(j=0,1, \ldots, N+1)$ and express

$$
\begin{aligned}
K= & g(|0\rangle\langle 1|+| N\rangle\langle N+1|+\text { H.c. }) \\
& +\sum_{j=1}^{N-1}(|j\rangle\langle j+1|+\text { H.c. }),
\end{aligned}
$$

where we have set the intrachain coupling strength $\kappa=1$. The time required for eigenmode-mediated state transfer is $t=$
$\sqrt{N+1} \pi / 2 g$ and $M=\exp (-i K t)$. Let us now define $| \pm\rangle=$ $(|0\rangle \pm|N+1\rangle) / \sqrt{2}$ and further suppose that $N=4 x-3$ for $x \in \mathbb{Z}_{>0}$ (we will consider the other case below). After going into the diagonal basis $|k\rangle(k=1, \ldots, N)$ of the intermediate chain, the Hamiltonian breaks down into two decoupled parts as follows:

$$
\begin{gathered}
K=K_{+}+K_{-}, \\
K_{-}=\sum_{k=\text { even }}\left[\Delta_{k}|k\rangle\langle k|+\Omega_{k}(|-\rangle\langle k|+\text { H.c. })\right], \\
K_{+}=\sum_{k=\text { odd }}\left[\Delta_{k}|k\rangle\langle k|+\Omega_{k}(|-\rangle\langle k|+\text { H.c. })\right],
\end{gathered}
$$

where $\quad \Delta_{k}=2 \cos [\pi k /(N+1)] \quad$ and $\quad \Omega_{k}=$ $(2 g / \sqrt{N+1}) \sin [\pi k /(N+1)]$. First consider $K_{-}$, which deals only with even $k$ and does not deal with the zero-energy mode $|z\rangle \equiv|(N+1) / 2\rangle$. The eigenstates are perturbed only slightly from the original states and we call them $|\tilde{-}\rangle$ and $|\tilde{k}\rangle$ with energy 0 and $\Delta_{k}+O\left(g^{2}\right)$, respectively. Moreover, we have

$$
|-\rangle \approx\left[1-\sum_{k}\left(\frac{\Omega_{k}}{\Delta_{k}}\right)^{2}\right]|\tilde{-}\rangle-\sum_{k} \frac{\Omega_{k}}{\Delta_{k}}|\tilde{k}\rangle .
$$

Thus

$$
\left\langle-\left|e^{-i K_{-} t}\right|-\right\rangle \approx 1-2 \sum_{k<z}\left(\frac{\Omega_{k}}{\Delta_{k}}\right)^{2}\left[1-\cos \left(\Delta_{k} t\right)\right],
$$

where, as discussed, the sum here is only over even $k$.

We now consider $K_{+}$, which deals with odd $k$ and is a little more difficult to treat since it has the zero-energy mode. The eigenstates are $|\tilde{s}\rangle$ (for symmetric) with energy $\Omega+O\left(g^{3}\right)$, $|\tilde{a}\rangle$ (for antisymmetric) with energy $-\Omega+O\left(g^{3}\right)$, and $|\tilde{k}\rangle$ (for all odd $k$ except for $k=z$ ) with energy $\Delta_{k}+O\left(g^{2}\right)$. We find

$$
|+\rangle \approx \frac{1}{\sqrt{2}}\left[1-\sum_{k<z}\left(\frac{\Omega_{k}}{\Delta_{k}}\right)^{2}\right](|\tilde{s}\rangle+|\tilde{a}\rangle)-\sum_{k \neq z} \frac{\Omega_{k}}{\Delta_{k}}|\tilde{k}\rangle .
$$

Thus

$$
\left\langle+\left|e^{-i K_{+} t}\right|+\right\rangle \approx-1+2 \sum_{k<z}\left(\frac{\Omega_{k}}{\Delta_{k}}\right)^{2}\left[1+\cos \left(\Delta_{k} t\right)\right],
$$

where the sum is only over odd $k$.

Putting the results together, we obtain

$$
\begin{aligned}
1 & -|\langle 0|M| N+1\rangle|^{2} \\
& =1-\frac{1}{4}\left|\left\langle+\left|e^{-i K_{+} t}\right|+\right\rangle-\left\langle-\left|e^{-i K_{-} t}\right|-\right\rangle\right|^{2} \\
& \approx 1-\frac{1}{4}\left[-2+2 \sum_{k<z}\left(\frac{\Omega_{k}}{\Delta_{k}}\right)^{2}\left[1-(-1)^{k} \cos \left(\Delta_{k} t\right)\right]\right]^{2} \\
& \approx 2 \sum_{k<z}\left(\frac{\Omega_{k}}{\Delta_{k}}\right)^{2}\left[1-(-1)^{k} \cos \left(\Delta_{k} t\right)\right],
\end{aligned}
$$




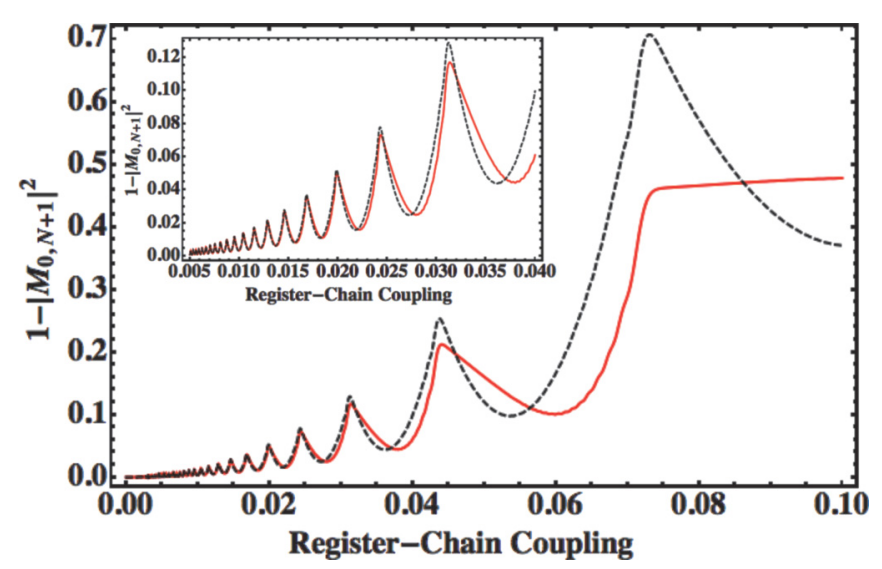

FIG. 10. (Color online) Comparison between the perturbative (dotted) and exact (solid) calculations for the matrix element $M_{0, N+1}$ for $N=205$. Here $1-\left|M_{0, N+1}\right|^{2}$ is plotted as a function of $g$, the register-chain coupling strength $(\kappa=1)$. As expected, perturbation theory breaks down as $g \sim \kappa / \sqrt{N}$. The inset depicts a close-up of the region for $0.005<g<0.04$, where the perturbative expression is in excellent agreement with the exact calculation.

where the sum is over both odd and even $k$ less than $z \equiv$ $(N+1) / 2$. Generalizing to all odd $N$, we find

$1-|\langle 0|M| N+1\rangle|^{2} \approx 2 \sum_{k<z}\left(\frac{\Omega_{k}}{\Delta_{k}}\right)^{2}\left[1+(-1)^{k+z} \cos \left(\Delta_{k} t\right)\right]$

where $z=(N+1) / 2$ and the sum is over both odd and even $k$ (Fig. 10).

Let us now generalize our expression to include the case of even $N$. Keeping $\Delta_{k}=2 \cos [\pi k /(N+1)]$ and $\Omega_{k}=$ $(2 g / \sqrt{N+1}) \sin [\pi k /(N+1)]$, we now have $z=N / 2, t=$ $\pi / \Omega_{z}$, and states 0 and $N+1$ are shifted by $\Delta_{z}+\delta$ [note that $\left.\delta \sim O\left(g^{2}\right)\right]$. We now define $\tilde{\Delta}_{k}=\Delta_{k}-\Delta_{z}$ and shift all energies by $\Delta_{z}$ so that $|0\rangle$ and $|N+1\rangle$ are now at energy $\delta$. Since we are interested in the square of the matrix element, this overall energy shift will not affect our result. We take $K_{ \pm}$ and $| \pm\rangle$ as before. As before, we first consider $N=4 x$ for $x \in \mathbb{Z}_{>0}$ (the remaining even $N$ will be discussed below).

We calculate the evolution of $|+\rangle$, which is coupled to all odd $|k\rangle$ and thus not coupled to $|z\rangle$. We have

$$
\begin{aligned}
e^{-i K_{+} t}|+\rangle \approx & {\left[1-\frac{1}{2} \sum_{k}\left(\frac{\Omega_{k}}{\tilde{\Delta}_{k}}\right)^{2}\right]|\tilde{+}\rangle e^{i A} } \\
& -\sum_{k} \frac{\Omega_{k}}{\tilde{\Delta}_{k}}|\tilde{k}\rangle e^{-i \tilde{\Delta}_{k} t}
\end{aligned}
$$

where $A=-\delta t+\sum_{k} \frac{\Omega_{k}^{2}}{\tilde{\Delta}_{k}} t$. To second order,

$$
\left\langle+\left|e^{-i K_{+} t}\right|+\right\rangle \approx e^{i A}-\sum_{k}\left(\frac{\Omega_{k}}{\tilde{\Delta}_{k}}\right)^{2}\left[1-\cos \left(\tilde{\Delta}_{k} t\right)\right],
$$

where the sum is over odd $k$. We now consider the evolution of $|-\rangle$ (which is coupled to all even $|k\rangle$, including $|z\rangle$ ). We find

$$
\begin{aligned}
e^{-i K_{-} t}|-\rangle \approx & \frac{1}{\sqrt{2}}\left[1-\frac{1}{2} \sum_{k \neq z}\left(\frac{\Omega_{k}}{\tilde{\Delta}_{k}}\right)^{2}\right](|\tilde{s}\rangle+|\tilde{a}\rangle)(-1) e^{i B} \\
& -\sum_{k \neq z} \frac{\Omega_{k}}{\tilde{\Delta}_{k}}|\tilde{k}\rangle e^{-i \tilde{\Delta}_{k} t}
\end{aligned}
$$

where $B=\left(-\delta t+\sum_{k} \frac{\Omega_{k}^{2}}{\tilde{\Delta}_{k}} t\right) / 2$. Thus

$$
\left\langle-\left|e^{-i K_{-} t}\right|-\right\rangle \approx-e^{i B}+\sum_{k \neq z}\left(\frac{\Omega_{k}}{\tilde{\Delta}_{k}}\right)^{2}\left[1+\cos \left(\tilde{\Delta}_{k} t\right)\right],
$$

where the sum is over even $k$. Putting the calculations together, we find

$$
\begin{aligned}
1 & -|\langle 0|M| N+1\rangle|^{2} \\
& =1-\frac{1}{4}\left|\left\langle+\left|e^{-i K_{+} t}\right|+\right\rangle-\left\langle-\left|e^{-i K_{-} t}\right|-\right\rangle\right|^{2} \\
& \approx \sum_{k \neq z}\left(\frac{\Omega_{k}}{\tilde{\Delta}_{k}}\right)^{2}\left[1+(-1)^{k} \cos \left(\tilde{\Delta}_{k} t\right)\right]+\frac{1}{4}(A-B)^{2} .
\end{aligned}
$$

Generalizing to all even $N$, we have

$$
\begin{aligned}
1-|\langle 0|M| N+1\rangle|^{2} \approx & \sum_{k \neq z}\left(\frac{\Omega_{k}}{\tilde{\Delta}_{k}}\right)^{2}\left[1+(-1)^{k+z} \cos \left(\tilde{\Delta}_{k} t\right)\right] \\
& +\frac{1}{4}(A-B)^{2},
\end{aligned}
$$

where

$$
\begin{aligned}
& A=\frac{3+(-1)^{z}}{4}\left[-\delta t+\sum_{\operatorname{odd} k \neq z} \frac{\Omega_{k}^{2}}{\tilde{\Delta}_{k}} t\right], \\
& B=\frac{3-(-1)^{z}}{4}\left[-\delta t+\sum_{\text {even } k \neq z} \frac{\Omega_{k}^{2}}{\tilde{\Delta}_{k}} t\right] .
\end{aligned}
$$

Thus, by setting

$$
\delta=\sum_{k \neq z} \frac{1-3(-1)^{z+k}}{2} \frac{\Omega_{k}^{2}}{\tilde{\Delta}_{k}}
$$

we obtain $A-B=0$, yielding

$$
1-|\langle 0|M| N+1\rangle|^{2} \approx \sum_{k \neq z}\left(\frac{\Omega_{k}}{\tilde{\Delta}_{k}}\right)^{2}\left[1+(-1)^{k+z} \cos \left(\tilde{\Delta}_{k} t\right)\right]
$$

which holds for both even and odd $N$. One should note that tuning $\delta$ only affects small $N$ since for larger $N,(A-B)^{2} / 4$ is negligible.

We now compute $M_{0,0}$ by employing the techniques outlined above; moreover, we note that any requisite matrix element entering the average channel fidelity formulas can be computed in a similar fashion. For the $M_{0,0}$ case, the $K_{-}$ expression remains identical to Eq. (A6). However, the $K_{+}$ expression [Eq. (A8)] is now different,

$$
\left\langle+\left|e^{-i K_{+} t}\right|+\right\rangle \approx 1-2 \sum_{k<z}\left(\frac{\Omega_{k}}{\Delta_{k}}\right)^{2}\left[1-\cos \left(\Delta_{k} t\right)\right]
$$


where the sum is over odd $k$. Combining yields

$$
\begin{aligned}
1-M_{0,0} & =1-\frac{1}{2}\left[\left\langle+\left|e^{-i K_{+} t}\right|+\right\rangle+\left\langle-\left|e^{-i K_{-} t}\right|-\right\rangle\right] \\
& =\sum_{k<z}\left(\frac{\Omega_{k}}{\Delta_{k}}\right)^{2}\left[1-\cos \left(\Delta_{k} t\right)\right],
\end{aligned}
$$

where the sum is now over both odd and even $k$.

\section{APPENDIX B: CHANNEL FIDELITY FOR THE REMOTE $\sigma^{z}$ GATE}

In this Appendix we illustrate the channel fidelity associated with an eigenmode-mediated remote $\sigma^{z}$ gate. In combination with the detailed discussion of the double-swap channel fidelity in Sec. III, this provides the framework for calculating the gate fidelity of a remote controlled-PHASE gate. In particular, we examine the process whereby (i) register 0 is swapped across the intermediate chain, (ii) a $\sigma^{z}$ gate is performed at register $N+1$, and (iii) a second return step of eigenmode-mediated state transfer is performed. In the ideal case, this remote $\sigma^{z}$ channel should result in a $\sigma^{z}$ gate on register 0 and hence the associated fidelity is given by

$$
F_{z}=\frac{1}{2}+\frac{1}{12} \sum_{i=x, y, z} \operatorname{Tr}_{0}\left\{\sigma_{0}^{z} \sigma_{0}^{i} \sigma_{0}^{z} \operatorname{Tr}_{A}\left[U_{z}\left(\sigma_{0}^{i} \otimes \rho_{\mathrm{ch}}\right) U_{z}^{\dagger}\right]\right\}
$$

where $U_{z}=U \sigma_{N+1}^{z} U, U$ represents an eigenmode-mediated swap, $\rho_{\text {ch }}$ is the mixed state of spins $1, \ldots, N+1, \operatorname{Tr}_{0}$ traces over register 0 , and $\operatorname{Tr}_{A}$ traces over all other spins. Let us begin by calculating the time evolution of $\sigma_{0}^{+}$,

$$
\begin{aligned}
\sigma_{0}^{+}(t) & =U_{z}^{\dagger} c_{0}^{\dagger} U_{z} \\
& =U^{\dagger} e^{i \pi n_{N+1}} U^{\dagger} c_{0}^{\dagger} U e^{i \pi n_{N+1}} U \\
& =U^{\dagger}\left[M_{0,0}^{*} c_{0}^{\dagger}-M_{0, N+1}^{*} c_{N+1}^{\dagger}+\sum_{i} M_{0, i}^{*} c_{i}^{\dagger}\right] U \\
& \rightarrow\left(M_{0,0}^{*}\right)^{2} c_{0}^{\dagger}-\left(M_{0, N+1}^{*}\right)^{2} c_{N+1}^{\dagger}+\sum_{i}\left(M_{0, i}^{*}\right)^{2} c_{i}^{\dagger},
\end{aligned}
$$

where we have used the fact that the number of excitations in each mode must be preserved. As before, for $i=x, y$, only cross terms involving $\sigma^{+}$and $\sigma^{-}$provide a nonzero contribution to Eq. (B1). We find

$$
\begin{aligned}
& \operatorname{Tr}_{0}\left\{\left[\sigma_{0}^{z} \sigma_{0}^{+} \sigma_{0}^{z} \operatorname{Tr}_{A}\left[U_{z}\left(\sigma_{0}^{-} \otimes \rho_{\mathrm{ch}}\right) U_{z}^{\dagger}\right]\right\}\right. \\
& \quad=\left(M_{0, N+1}^{*}\right)^{2}-\left(M_{0,0}^{*}\right)^{2}-\sum_{i}\left(M_{0, i}^{*}\right)^{2} .
\end{aligned}
$$

An analogous calculation yields the contribution from the opposite cross term and thus we now turn to the $\sigma^{z}$ contribution. Again, we begin by calculating the time evolution

$$
\begin{aligned}
\sigma_{0}^{z}(t) & =U^{\dagger} e^{i \pi n_{N+1}} U^{\dagger}\left(2 c_{0}^{\dagger} c_{0}-1\right) U e^{i \pi n_{N+1}} U \\
& \rightarrow 2 U^{\dagger} e^{i \pi n_{N+1}} \sum_{i, j} M_{0, i}^{*} M_{0, j} c_{i}^{\dagger} c_{j} e^{i \pi n_{N+1}} U,
\end{aligned}
$$

where we have dropped the $(-1)$ contribution from the first line since it will ultimately trace to zero. Conjugation by $e^{i \pi n_{N+1}}$ affects $\sum_{i, j} M_{0, i}^{*} M_{0, j} c_{i}^{\dagger} c_{j}$ only if $i$ or $j$ equals $N+1$; in these cases, the matrix element gets an additional negative sign. We can capture this by defining an $(N+2) \times(N+2)$ diagonal matrix $S$, which contains unity along all diagonal entries except the last, where it contains $(-1)$. Using $S$, we find

$$
\begin{aligned}
\sigma_{0}^{z}(t) & =2 U^{\dagger} \sum_{i, j} \tilde{M}_{0, i}^{*} \tilde{M}_{0, j} c_{i}^{\dagger} c_{j} U \\
& \rightarrow 2 \sum_{i, j} \tilde{M}_{0, i}^{*} \tilde{M}_{0, j} \sum_{i^{\prime}, j^{\prime}} M_{i, i^{\prime}}^{*} M_{j, j^{\prime}} c_{i^{\prime}}^{\dagger} c_{j^{\prime}},
\end{aligned}
$$

where $\tilde{M}=M S$. A nonzero contribution arises only if $i^{\prime}=$ $j^{\prime}=0$, wherein we find $\operatorname{Tr}\left[\sigma_{0}^{+} \sigma_{0}^{-} \sigma_{0}^{z}\right]=1$. Combining all contributions yields

$$
\begin{aligned}
F_{z}= & \frac{1}{2}+\frac{1}{6}\left[\left(M_{0, N+1}^{*}\right)^{2}-\left(M_{0,0}^{*}\right)^{2}-\sum_{i}\left(M_{0, i}^{*}\right)^{2}+\text { c.c. }\right] \\
& +\frac{1}{6} \sum_{i, j} \tilde{M}_{0, i}^{*} \tilde{M}_{0, j} M_{i, 0}^{*} M_{j, 0} \\
= & \frac{1}{2}+\frac{1}{6}\left[|\langle 0|M S M| 0\rangle|^{2}-2 \operatorname{Re}(\langle 0|M S M| 0\rangle)\right],
\end{aligned}
$$

where we have made use of the fact that $M$ is symmetric.
[1] C. H. Bennett, G. Bassard, C. Crépeau, R. Jozsa, A. Peres, and W. K. Wootters, Phys. Rev. Lett. 70, 1895 (1993).

[2] H. J. Kimble, Nature (London) 453, 1023 (2008).

[3] N. Y. Yao et al., Nat. Commun. 3, 800 (2012).

[4] T. D. Ladd et al., Nature (London) 464, 45 (2010).

[5] J. J. L. Morton et al., Nature (London) 455, 1085 (2008).

[6] A. M. Tyryshkin et al., Nature Materials 11, 143 (2012).

[7] T. D. Ladd, D. Maryenko, Y. Yamamoto, E. Abe, and K. M. Itoh, Phys. Rev. B 71, 014401 (2005).

[8] P. Maurer et al., Science 336, 1283 (2012).

[9] A. D. Greentree, J. H. Cole, A. R. Hamilton, and L. C. L. Hollenberg, Phys. Rev. B 70, 235317 (2004).

[10] B. B. Blinov et al., Nature (London) 428, 153 (2004).

[11] D. L. Moehring et al., Nature (London) 449, 68 (2007).
[12] E. Togan et al., Nature (London) 466, 730 (2010).

[13] J. Twamley and S. D. Barrett, Phys. Rev. B 81, 241202(R) (2010).

[14] C. Chudzicki and F. W. Strauch, Phys. Rev. Lett. 105, 260501 (2010).

[15] L.-A. Wu, A. Miranowicz, X.B. Wang, Y.-x. Liu, and F. Nori, Phys. Rev. A 80, 012332 (2009).

[16] M. B. Plenio et al., New J. Phys. 6, 36 (2004).

[17] M. Riebe et al., Nat. Phys. 4, 839 (2008).

[18] L. Banchi, A. Bayat, P. Verrucchi, and S. Bose, Phys. Rev. Lett. 106, 140501 (2011).

[19] B. E. King, C. S. Wood, C. J. Myatt, Q. A. Turchette, D. Leibfried, W. M. Itano, C. Monroe, and D. J. Wineland, Phys. Rev. Lett. 81, 1525 (1998). 
[20] M. A. Sillanpaa et al., Nature (London) 449, 438 (2007).

[21] J. Q. You and F. Nori, Nature (London) 474, 589 (2011).

[22] J. Majer et al., Nature (London) 449, 443 (2007).

[23] C. D. Ogden, E. K. Irish, and M. S. Kim, Phys. Rev. A 78, 063805 (2008).

[24] S. Bose et al., J. Mod. Opt. 54, 2307 (2007).

[25] G. D. de Moraes Neto, M. A. de Ponte, and M. H. Y. Moussa, Phys. Rev. A 84, 032339 (2011).

[26] S. Bose, Phys. Rev. Lett. 91, 207901 (2003).

[27] N. Y. Yao, L. Jiang, A. V. Gorshkov, Z.-X. Gong, A. Zhai, L.-M. Duan, and M. D. Lukin, Phys. Rev. Lett. 106, 040505 (2011).

[28] E. B. Fel'dman, E. I. Kuznetsova, and A. I. Zenchuk, Phys. Rev. A 82, 022332 (2010).

[29] G. Gualdi, V. Kostak, I. Marzoli, and P. Tombesi, Phys. Rev. A 78, 022325 (2008).

[30] D. Petrosyan, G. M. Nikolopoulos, and P. Lambropoulos, Phys. Rev. A 81, 042307 (2010).

[31] M. Christandl, N. Datta, A. Ekert, and A. J. Landahl, Phys. Rev. Lett. 92, 187902 (2004).

[32] D. Burgarth, V. Giovannetti, and S. Bose, Phys. Rev. A 75, 062327 (2007).

[33] C. Di Franco, M. Paternostro, and M. S. Kim, Phys. Rev. Lett. 101, 230502 (2008).

[34] A. Kay, Phys. Rev. Lett. 98, 010501 (2007).

[35] S. R. Clark et al., New J. Phys. 7, 124 (2005).

[36] A. Bayat, L. Banchi, S. Bose, and P. Verrucchi, Phys. Rev. A 83, 062328 (2011)

[37] A. Wójcik, T. Łuczak, P. Kurzyński, A. Grudka, T. Gdala, and M. Bednarska, Phys. Rev. A 72, 034303 (2005).

[38] P. Karbach and J. Stolze, Phys. Rev. A 72, 030301(R) (2005).

[39] L. Banchi, T. J. G. Apollaro, A. Cuccoli, R. Vaia, and P. Verrucchi, Phys. Rev. A 82, 052321 (2010).

[40] M. Bruderer et al., Phys. Rev. A 85, 022312 (2012).

[41] V. S. Malinovsky and D. J. Tannor, Phys. Rev. A 56, 4929 (1997).

[42] E. B. Fel'dman and A. I. Zenchuk, Phys. Lett. A 373, 1719 (2009).

[43] J. Fitzsimons and J. Twamley, Phys. Rev. Lett. 97, 090502 (2006).

[44] J. Fitzsimons, L. Xiao, S. C. Benjamin, and J. A. Jones, Phys. Rev. Lett. 99, 030501 (2007).

[45] P. Cappellaro, C. Ramanathan, and D. G. Cory, Phys. Rev. Lett. 99, 250506 (2007).
[46] J. Zhang, M. Ditty, D. Burgarth, C. A. Ryan, C. M. Chandrashekar, M. Laforest, O. Moussa, J. Baugh, and R. Laflamme, Phys. Rev. A 80, 012316 (2009).

[47] P. Cappellaro, L. Viola, and C. Ramanathan, Phys. Rev. A 83, 032304 (2011).

[48] F. Evers and A. D. Mirlin, Rev. Mod. Phys. 80, 1355 (2008).

[49] L. Childress et al., Science 314, 281 (2006).

[50] G. Balasubramanian et al., Nat. Mater. 8, 383 (2009).

[51] E. Rittweger et al., Nat. Photon. 3, 144 (2009).

[52] A. G. Fowler, A. M. Stephens, and P. Groszkowski, Phys. Rev. A 80, 052312 (2009).

[53] D. S. Wang, A. G. Fowler, and L. C. L. Hollenberg, Phys. Rev. A 83, 020302(R) (2011).

[54] L. Banchi et al., New J. Phys. 13, 123006 (2011).

[55] A. Zwick, G. A. Álvarez, J. Stolze, and O. Osenda, Phys. Rev. A 85, 012318 (2012).

[56] K. Kim et al., New J. Phys. 13, 105003 (2011).

[57] S. Mostame and R. Schutzhold, Phys. Rev. Lett. 101, 220501 (2008).

[58] P. Jordan and E. Wigner, Z. Phys. 47, 631 (1928).

[59] A. Y. Kitaev, Usp. Fiz. Nauk 171, 131 (2001).

[60] C. Nayak, S. Simon, A. Stern, M. Freedman, and S. Das Sarma, Rev. Mod. Phys. 80, 1083 (2008).

[61] M. Markiewicz and M. Wiesniak, Phys. Rev. A 79, 054304 (2009).

[62] M. Nielsen, Phys. Lett. A 403, 249 (2002).

[63] S.-B. Shim et al., Science 316, 95 (2007).

[64] K. R. Brown et al., Nature (London) 471, 196 (2010).

[65] F. S. Cataliotti et al., Science 293, 843 (2001).

[66] S. Teitel and C. Jayaprakash, Phys. Rev. Lett. 51, 1999 (1983).

[67] Y. Makhlin et al., Rev. Mod. Phys. 73, 357 (2001).

[68] A. H. Safavi-Naeini et al., Nature (London) 472, 69 (2011).

[69] D. Burgarth, Eur. Phys. J. Spec. Top. 151, 147 (2007).

[70] A. Zwick and O. Osenda, J. Phys. A 44, 105302 (2011).

[71] A. Jarmola, V. M. Acosta, K. Jensen, S. Chemerisov, and D. Budker, Phys. Rev. Lett. 108, 197601 (2012).

[72] P. Maurer et al., Nat. Phys. 6, 912 (2010).

[73] P. Spinicelli et al., New J. Phys. 13, 025014 (2011).

[74] D. M. Toyli et al., Nano Lett. 10, 3168 (2010).

[75] T. J. G. Apollaro et al., Phys. Rev. A 85, 052319 (2012).

[76] E. H. Lieb and D. W. Robinson, Commun. Math. Phys. 28, 251 (1972). 